\title{
Highlights of the Canadian Frailty Network (CFN) Highly Qualified Personnel Poster Session
}

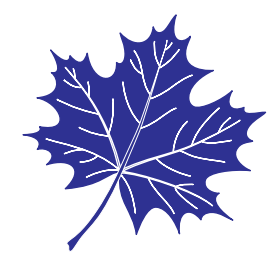

\author{
John Muscedere ${ }^{1}$, Amber Hastings Truelove ${ }^{2}$, Denise Stockley ${ }^{2}$, Jennifer Fowler ${ }^{2}$, Carol Barrie ${ }^{1}$ \\ ${ }^{I}$ Canadian Frailty Network, Kingston, ON, Canada; ${ }^{2}$ Queen's University, Kingston, ON, Canada
}

DOI:http://dx.doi.org/10.57700/cgj.22.369

The Canadian Frailty Network (CFN) was established in 2012 under the Canadian Networks of Centers of Excellence (NCE) program. The goal of the NCE Program is to mobilize Canada's research talent in the academic, private, public, and not-for-profit sectors, and apply it to the task of developing the economy and improving the quality of life of Canadians. As a national network, CFN is dedicated to improving the lives of older adults living with frailty and their family/friend caregivers.

We do this by increasing frailty recognition and assessment, increasing evidence for decision-making, advancing evidence-based changes to care, and engaging with older adults and caregivers. We are a multi-disciplinary network, nurturing productive multi-sectoral partnerships and collaborations, and always including patients, families, and caregivers.

A core part of our mandate from NCE is to provide training for the next generation of researchers, health-care practitioners, and policy-makers engaged in care for older Canadians living with frailty. Highly Qualified Personnel (HQP) enter the CFN Training Program as part of our Summer Student Award Program, our one-year Interdisciplinary Fellowship Program, or as research trainees attached to a project that has received CFN funding. Our goal is to develop Highly Qualified Personnel with the disciplinary, interdisciplinary, and transdisciplinary skills, experiences, and attitudes necessary to provide creative solutions for issues facing older adults living with frailty and their family/ friend caregivers. The program provides HQP with unique educational experiences that deepen appreciation for holistic care, increase exposure to interdisciplinary research through knowledge creation and translation projects, and advance intellectual and professional development.

One of the highlights of our training program is the opportunity our HQP have to disseminate their research to national and international experts on frailty. At the CFN 2018 National Conference-“"Improving Frailty Care Benefits Everyone"-held in Toronto, September 20-21, all 2017 and $2018 \mathrm{HQP}$ had the opportunity to present posters which showcased their projects to researchers, practitioners, educators, policy-makers, and advocates, as well as to citizen partners engaged in $\mathrm{CFN}$ research projects.

HQP submitted abstracts for their posters which were vetted and returned with commentary. The HQP had the opportunity to make revisions before making a final abstract submission. This year we had strong turnout from our HQP, in numbers and in poster quality, making our judges' job incredibly difficult. Posters were divided into 3 categories, Fellowship submissions, Summer Student Award submissions, and submissions from HQP attached to a CFN-funded research project. This ensured that our undergraduate students would not be competing against graduate students and postdoctoral fellows.

The winners from each category represent the outstanding scholarly contributions to frailty research being undertaken by our HQP, and are representative of the breadth of topics with which they engage:

- Outstanding poster by a Summer Student was awarded to Karl Grewal from the University of Victoria for the poster "A Community Choir to Facilitate Psychosocial and Cognitive Health for Caregivers and Persons with Dementia".

- Outstanding poster by an Interdisciplinary Fellow was awarded to Elaine Moody from Dalhousie University for the poster "Exploring the Needs and Associated Out-ofPocket Expenses of Older People Living with Frailty who Wish to Age-in-Place".

- Outstanding poster presented by a trainee on a CFNFunded Project was awarded to Abe Hafid from McMaster University for the poster "Advance Care Planning: Building Capacity in Interprofessional Primary Care Teams".

In what follows, we present the compilation of research abstracts that were presented by CFN HQP at the $2018 \mathrm{Na}-$ tional Conference. 


\section{Advance Care Planning: Building Capacity in Inter- professional Primary Care Teams}

A. Hafid, D. Guenter, E. Gallagher, M. Howard, J. You, A. Nidumolu. Department of Family Medicine, McMaster University, Hamilton, ON, Canada.

Background: Most adults prefer that their health-care providers initiate advance care planning (ACP) conversations. Yet, primary care providers (PCPs) rarely discuss ACP with patients. The goal of this study is to implement and evaluate communication training for PCPs to improve the quantity and quality of ACP conversations.

Methods: This is a quality improvement project in a large interprofessional academic family medicine team. PCPs $(\mathrm{n}=$ 30) attended 2.5-hour Serious Illness Conversation Guide (SICG) training workshops, adapted from Ariadne Labs, to improve their ACP capabilities. Participants completed preand post-workshop self-assessments. PCPs will use the SICG to conduct ACP with 3 patients each. Eligible patients are aged 65 or older, having any diagnosis of chronic illness or frailty that shortens lifespan. PCPs and patients will complete questionnaires gauging their experiences.

Results: Post-workshop assessments reported improvements in confidence, prognosis sharing, and exploring patient values and trade-offs. PCPs reported the workshops as highly effective in improving ACP skills. Results to be presented will also include PCP, patient, and family member ACP satisfaction.

Conclusions: Results will inform adaptations for further primary care training and implementation strategies.

\section{The Effects of the Serious Illness Care Program (SICP) on Health Care Resource Utilization (HRU)}

A. Lagrotteria, J. You. McMaster University, Hamilton, ON, Canada.

Objective: The Serious Illness Care Program (SICP)—a structured communication intervention-aims to improve the quality of goals of care conversations for hospitalized patients who are frail. We hypothesized that the SICP may reduce frail patients' length of stay (LOS) and intensity of treatment, and therefore be associated with differences in health resource utilization (HRU) compared to usual care.

Methods: In this retrospective cohort study, we assessed HRU within 60 days following the index admission of eligible patients (admitted $>48$ hours to a medical ward and an Assessment
Urgency Algorithm Score of 5 or 6, i.e., frail) enrolled into the SICP or who received usual care.

Results: There was no difference for acute (non-alternate level of care [ALC]) LOS between control and SICP patients (17 vs. 18.5 days, $p=0.59$ ). However, analysis of readmissions within 60 days revealed that SICP patients had shorter acute (non-ALC) LOS versus usual care (6 vs. 18.5 days, $p=0.01$ ). We observed no other differences in HRU between SICP and usual care.

Conclusion: In a hospital medical ward setting, the SICP was associated with reduced acute (non-ALC) LOS on readmission to hospital. An increased sample size and longer follow-up may enable greater inferences.

\section{Anticipatory Long-Term Care Resident Triage (ALERT) Tool: Lessons Learned from Trial of Implementation of e-INTERACT in 4 Canadian Long-Term Care Homes}

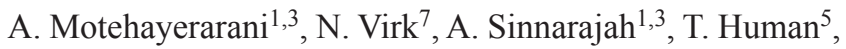
I. Ying ${ }^{4}$, H. Wong 6 , G. Cummings ${ }^{2,3}$, A. Pattullo ${ }^{1,3}$, J. Wang ${ }^{8}$, J. Lee ${ }^{1}$, D. Moffat ${ }^{8}$, M. Grinman ${ }^{1,3}$. ${ }^{1}$ University of Calgary, Calgary, AB, Canada; ${ }^{2}$ University of Alberta, Edmonton AB, Canada; ${ }^{3}$ Alberta Health Services, Edmonton, AB, Canada; ${ }^{4}$ University of Toronto, Toronto, ON, Canada; ${ }^{5}$ Palliative Pain and Symptom Management Consultation Service, Toronto, ON, Canada; ${ }^{6}$ York University, Toronto, ON, Canada; ${ }^{7}$ Brenda Strafford Foundation, Calgary, AB, Canada; ${ }^{8}$ Sienna Senior Living Foundation, Markham, ON, Canada.

Background: e-INTERACT, a set of tools for long-term care homes (LTCHs) in the United States, proactively identifies frail residents at risk of Emergency Department (ED) transfers and health status decline, to reduce potentially avoidable hospitalizations associated with increased risk of morbidity and mortality. This study assessed feasibility of implementing e-INTERACT in Canadian LTCHs.

Methods: e-INTERACT was implemented in 4 Canadian LTCHs in a 6-month pilot study. Four clinicians reviewed 645 encounters for 117 residents to determine whether e-INTERACT alerts should have been generated and their potential impact on goals of care discussions and ED transfers. Staff and resident surveys, and thematic analysis of interviews/focus groups identified facilitators and barriers for using the tools.

Results: Minimal uptake of e-INTERACT tools impeded validation for clinical use in Canada; frontline staff cited competing demands and a desire to communicate directly with their superiors. However, the research team identified that, in the majority of cases, regular use of e-INTERACT could have identified clinically meaningful patterns to prompt a more palliative approach to care. 
Conclusion: Implementation of e-INTERACT in Canada requires an assessment of LTCH culture and workflow integration to remove barriers to its use.

Implementation of the Clinical Frailty Scale into the Alberta Intensive Care Unit Electronic Medical Record

C. L. Montgomery ${ }^{1,2,6}$, D. B. Rolfson ${ }^{2,3}$, H. T. Stelfox ${ }^{4,5}$, D. Zuege $^{4,5}$, D. A. Zygun ${ }^{1,5}$, D. Hudson ${ }^{1}$, D. Opgenorth ${ }^{1}$, S. M. Bagshaw ${ }^{1,2,5} .{ }^{1}$ Department of Critical Care Medicine, University of Alberta, Edmonton, AB, Canada; ${ }^{2}$ Department of Medicine, University of Alberta, Edmonton, AB, Canada; ${ }^{3}$ Division of Geriatric Medicine, Faculty of Medicine \& Dentistry, University of Alberta, Edmonton, AB, Canada; ${ }^{4}$ Department of Critical Care Medicine, Cumming School of Medicine, University of Calgary, Calgary, AB, Canada; ${ }^{5}$ Alberta Health Services, Edmonton, AB, Canada; ${ }^{6}$ Canadian Frailty Network Interdisciplinary Fellow, Canadian Frailty Network, Kingston, ON, Canada.

Background: Frailty is common although rarely screened for in ICU settings. The population epidemiology of frailty associated with critical illness has yet to be studied. In 2016 the Clinical Frailty Scale (CFS) was incorporated as a routine measure into the provincial ICU electronic health record (eCritical Alberta) across Alberta adult ICUs.

Methods: An overview of frailty and associated outcomes (mortality and health resource utilization) was evaluated from 19,556 ICU admissions in the initial 18 months following the provincial CFS implementation.

Results: Of patients assigned a CFS score $(\mathrm{n}=15,238)$, mean age (SD) was $58( \pm 17)$ years, $39 \%$ were female, and $28 \%$ scored as frail (CFS $\geq 5$ ). Compared to nonfrail patients, those with frailty were more likely to be older, female, and medical admissions, with significantly higher SOFA and APACHE II scores. Frail admissions had significantly longer median duration of stay in ICU (4 vs. 3 days) and hospital (16 vs. 10 days), as well as higher ICU ( $12 \%$ vs. $7 \%$ ) and hospital mortality ( $25 \%$ vs. $10 \%$ ), respectively (all comparisons, $p<0.001$ ).

Conclusion: Further research is needed to incorporate frailty assessment into novel interventions to address those patients who are at greater risk for death and additional health resource utilization.

\section{Strategies for Sustaining and Spreading Improved Nutrition Care in Hospital}

C. Laur ${ }^{1,3}$, J. Bell ${ }^{4}$, R. Valaitis ${ }^{1}$, S. Ray ${ }^{3}$, H. Keller ${ }^{1,2} .{ }^{1}$ University of Waterloo, Waterloo, ON, Canada; ${ }^{2}$ Schlegel-University of Waterloo Research Institute for Aging, Waterloo, ON, Canada $;{ }^{3}$ NNEdPro Global Centre for Nutrition and Health, Cambridge, United Kingdom; ${ }^{4}$ The University of Queensland $\&$ The Prince Charles Hospital, Brisbane, Australia.

Background: Targeted and effective strategies are needed to sustain and spread positive changes in health care. This study aimed to develop an understanding of hospital staff/management perspectives regarding sustaining and spreading successful nutrition care practice changes to benefit the frail elderly.

Methods: The More-2-Eat project used participatory action research to implement a best practice algorithm for nutrition care in five medical units in four provinces. After implementation, key informant interviews (KI) were conducted with hospital management and focus groups (FG) with staff. Results were thematically analysed.

Results: In 2016, KI ( $\mathrm{n}=45)$ and FG (15 FG, $\mathrm{n}=78)$ were completed. A year later, interviews $(\mathrm{n}=12)$ were conducted with key staff. Themes focused on what changed and how improvements were sustained and spread throughout the unit/ hospital. Strategies to sustain changes included: maintaining the routine; building intrinsic motivation; continuing to collect and share data (audits); and engaging new staff and management. Strategies to spread changes included: making it easy; being responsive to opportunities; and considering local context and readiness. Strategies for both included: being and staying visible; and maintaining roles and supporting new champions.

Conclusion: Themes identified strategies that led to a culture of nutrition care that encouraged lasting positive impact, particularly for frail elderly.

\section{Resistance Training to Improve Physical Function in Pre-Frail Adults: A Randomized Trial}

C. Prevett ${ }^{1}$, H. Fang ${ }^{1}$, D. Shkredova ${ }^{1}$, F. Xie ${ }^{2}$, M. Zoratti ${ }^{2}$, C. Gordon ${ }^{3}$, J. Adachi ${ }^{3}$, S. Phillips ${ }^{4}$, J. Richardson ${ }^{1}$, A. Tang. ${ }^{1}$ School of Rehabilitation Science, McMaster University, Hamilton, ON, Canada; ${ }^{2}$ Department of Clinical Epidemiology and Biostatistics, McMaster University, Hamilton, ON, Canada; ${ }^{3}$ Department of Medicine, McMaster University, Hamilton, ON, Canada; ${ }^{4}$ Department of Kinesiology, McMaster University, Hamilton, ON, Canada.

Background: Resistance training (RT) is a promising strategy in preventing pre-fail patients from becoming frail. This study: 1) Examined safety and feasibility of high-intensity RT (HIRT); and 2) Compared effects of HI-RT to low-intensity RT (LO-RT) on functional outcomes in pre-frail persons.

Methods: Pre-frail individuals (74.6 \pm 7.4 years) performed 12 weeks of twice-weekly HI-RT $(3$ sets $\times 3-5$ repetitions, 
intensity 6-8/10) or LO-RT exercise (3 sets $\times 10-15$ repetitions, intensity 4-5/10) in this assessor-blinded randomized trial. Feasibility was evaluated by program adherence. Strength, endurance, and balance were assessed at baseline, after the 12-week program, and 8-week follow-up. Information on health-care utilization was collected.

Results: There were no adverse events related to interventions. No differences were observed between groups over time, however both interventions improved in 6 minute-walk distance (332.4 $\mathrm{m} \pm 81.6$ to $385.9 \mathrm{~m} \pm 106.5$ to $377.5 \mathrm{~m} \pm 91.8 ; p=0.03$ ), Berg Balance Scale $(50.4 \pm 4.2$ to $52.5 \pm 3.1$ to $52.4 \pm 3.5$; $p=0.03)$, and Timed Up and Go $(10.2 \mathrm{~s} \pm 2.6$ to $8.9 \mathrm{~s} \pm 1.8$ to $8.6 \mathrm{~s} \pm 2.1 ; p=0.02$ ) immediately following the intervention and at eight-week follow. Limited data prevented full health-care utilization economic analysis.

Conclusion: HI-RT is safe and feasible in pre-frail individuals. Future research is warranted to determine ideal RT parameters and health-care economic analysis.

\section{Assessing Quality of Goals of Care Conversations During SICP QI Initiative}

C. Ma*, L. Riehm*, J. You. Department of Medicine, McMaster University, Hamilton, ON, Canada.

*Contributed equally to this work

Background: Seriously ill and frail inpatients have indicated that better goals-of-care conversations improve end-of-life care. The Serious Illness Care Program (SICP) is an ongoing quality improvement initiative at Hamilton General Hospital (HGH). Previous work has shown that the SICP improves goals-ofcare conversations. This study assessed whether goals-of-care conversations have improved after implementation of the SICP.

Methods: This retrospective chart review evaluated patients admitted to medical wards at HGH. Eligible patients were admitted for at least 48 hours and had an Assessment Urgency Algorithm score of 5 or 6 (indicating frailty). The quality of documented goals-of-care conversations were assessed using a validated codebook on the following domains: patients' values/goals, prognosis/illness understanding, end-of-life care planning, and code status.

Results: The study sample included 50 control patients from the pre-SICP time period and 50 patients from the SICP period. Full ethics approval was obtained. Results will include baseline characteristics and a comparison of quality of goals-ofcare conversations between control and intervention groups.

Conclusions: We hypothesize that the quality of goals-of-care conversations will have improved since SICP implementation.
If confirmed, this study will demonstrate the benefits of a hospital-based implementation of the SICP to improve goalsof-care conversations.

\section{Engaging Primary Health-Care Providers in Advance Care Planning: Recommendations from Stakeholder Dialogues}

C. Kendell ${ }^{1}$, R. Urquhart ${ }^{2}$, F. Burge ${ }^{3}$, J. Kotecha ${ }^{4}$, M. Mar$\operatorname{tin}^{4}$, M. Jorgensen ${ }^{1}$, H. Han ${ }^{4} .{ }^{1}$ Cancer Outcomes Research Program, Department of Surgery, Dalhousie University/Nova Scotia Health Authority, Halifax, NS, Canada; ${ }^{2}$ Department of Surgery, Dalhousie University, Halifax, NS, Canada; ${ }^{3}$ Department of Family Medicine, Dalhousie University, Halifax, NS, Canada; ${ }^{4}$ Department of Family Medicine, Queen's University, Kingston, ON, Canada.

Background: Advance care planning is vital to improving care for individuals with life limiting illness, however, advance care plans are often not in place when needed. Primary care providers are well situated to facilitate advance care planning and help ensure that seriously ill, frail older adults have an advance care plan in place.

Methods: Using the McMaster Health Forum approach, we carried out two stakeholder dialogues (Halifax and Kingston). The objective was to develop recommendations to improve the capacity of primary health-care providers to (1) identify patients who would benefit from a palliative approach to care and (2) engage in timely advance care planning. Participants were predominantly clinicians and administrators with expertise in primary, palliative, and long-term/continuing care $(n=22)$. Dialogues were one day long and comprised of several focused deliberations, culminating in the development and prioritization of actionable recommendations.

Results: The top recommendation from both dialogues was the creation of a designated, community-based role to support primary health-care providers and patients in the process of advance care planning (i.e., a "liaison").

Conclusion: Participants viewed primary health-care providers as capable and well situated for advance care planning, but also as lacking the time required to meaningfully engage patients in this process.

\section{Advance Care Planning with Older Gay Men Living with HIV: Challenges and Opportunities}

D. Dubé ${ }^{1}$, G. Gutman ${ }^{2}$, T. Sussman 1 , B. DeVries ${ }^{3}$, J. Gahagan ${ }^{4}$, S. Brotman ${ }^{1}$. School of Social Work, McGill University, 
Montreal, QC, Canada; ${ }^{2}$ Department of Gerontology, Simon Fraser University, Vancouver, BC, Canada; ${ }^{3}$ Department of Gerontology, San Francisco State University, San Francisco, CA, United States of America; ${ }^{4}$ School of Health and Human Performance, Dalhousie University, Halifax, NS, Canada.

Background: There is a growing awareness of the importance of engaging in early discussions about end-of-life care with older persons living with non-reversible chronic conditions. Such discussions have shown promise in reducing invasive treatments at end-of-life, supporting the delivery of holistic end-of-life care and improving care satisfaction for older adults, their families and health providers (Cornally et al., 2015; De Roo et al., 2010; van Soest-Poortvliet et al., 2015).

Methods: As a component of a national study focused on increasing the uptake, impact, and access to advance care planning (ACP) tools across the continuum of care (iCANACP), this study addresses the critical gap among older gay men aging with HIV. Using a focus group discussion and individual interview format with ten gay men 55+ in Montreal, we explored their perceptions, experiences, and barriers to engaging in ACP. Their preliminary reactions and use of the 'Conversation Starter Kit,' a workbook aimed at activating early end-of-life care discussions, was also explored.

Potential impact: This Study will deepen our understanding of gay men aging with HIV regarding early end-of-life discussions and ACP and allow improvement of access to ACP and better end of life care for this vulnerable population.

Pilot Study of an Automated One-Year Mortality Prediction Tool to Trigger Advance Care Planning

E. Koo ${ }^{1}$, P. Wegier ${ }^{2}$, G. Embuldeniya ${ }^{1}$, S. Ansari ${ }^{1}$, D. Kobe$\mathrm{wka}^{3}$, E. O'Connor ${ }^{1}$, P. Wu ${ }^{1}$, L. Steinberg ${ }^{2}$, C. Bell ${ }^{2}$, T. Walton $^{4}$, J. Colstello ${ }^{1}$, C. van Walraven ${ }^{3}$, J. Downar ${ }^{1} .{ }^{1}$ University Health Network, Toronto, ON, Canada; ${ }^{2}$ Sinai Health Systems, Toronto, ON, Canada; ${ }^{3}$ University of Ottawa, Ottawa, ON, Canada; ${ }^{4}$ Ontario Palliative Care Network Secretariat, Toronto, ON, Canada.

Background: The inability of clinicians to reliably and accurately identify patients with shortened life expectancy is an obstacle to improving palliative and end-of-life care (PEOLC).

Methods: An automated prospective clinical surveillance tool was used at two sites to identify patients with elevated mortality risk in this two phase mixed methods study. We abstracted 100 consecutive medical records of identified patients at each site, and compared the incidence of early $(<72 \mathrm{~h})$ goals of care (GOC) discussion and palliative care (PC) consultation between the two phases. Qualitative methods were used to assess the acceptability of the tool.

Results: At one site, identified patients were more likely to have an early GOC discussion or PC consultation (35\% vs. $20 \%, p=0.02$ ), while there was no significant difference at the other site. Some physicians found the prompts helpful, while others expressed concerns of redundancy or frequency. Patients and their family were generally well-disposed towards the tool, hoping it would prompt more communication from physicians.

Conclusions: We found that our application was a feasible and acceptable prospective clinical surveillance tool for patients with elevated mortality risk, and that the tool may be effective for improving PEOLC.

Using an Automated Mortality Prediction Tool to Focus Advance Care Planning Efforts for Inpatients: What are the Unmet Palliative Care Needs of Those Identified by the HOMR Application?

E. Koo ${ }^{1}$, P. Wegier ${ }^{2}$, S. Ansari ${ }^{1}$, D. Kobewka ${ }^{3}$, E. O'Connor ${ }^{1}$, P. E. Wu ${ }^{1}$, L. Steinberg ${ }^{2}$, C. Bell ${ }^{2}$, T. Walton ${ }^{4}$, J. Costello ${ }^{1}$, R. Wu ${ }^{1}$, D. Frost ${ }^{1}$, S. Kawaguchi ${ }^{2}$, R. Mahtani ${ }^{2}$, H. Toor ${ }^{2}$, R. Goldman ${ }^{2}$, J. Myers ${ }^{2}$, A. Forster ${ }^{3}$, C. van Walraven ${ }^{3}$, J. Downar ${ }^{1} .{ }^{1}$ University Health Network, Toronto, ON, Canada; ${ }^{2}$ Sinai Health Systems, Toronto, ON, Canada; ${ }^{3}$ University of Ottawa, Ottawa, ON, Canada; ${ }^{4}$ Ontario Palliative Care Network Secretariat, Toronto, ON, Canada.

Background: HOMR is an automated mortality prediction tool that identifies patients with elevated mortality risk. Patients who are frail and approaching end-of-life often have increasing symptom burden and should engage in advance care planning (ACP). However, the prevalence of unmet palliative needs among inpatients at risk of death (identified by HOMR) and readiness to engage in ACP is unknown.

Methods: We assessed the prevalence of unmet palliative needs among patients identified by HOMR by surveying 200 medicine admissions at two health-care facilities that use HOMR. We surveyed for uncontrolled symptoms and readiness to engage in $\mathrm{ACP}$, and compared the prevalence and severity of these unmet needs to those of patients identified by common, provider-dependent triggers (eg. CARENET criteria).

Results: Preliminary results show 69\% (38/55) of surveyed patients scored greater than 6/10 on the Edmonton Symptom Assessment System, indicating a "severe" symptom and unmet palliative need, while $65 \%$ (36/55) indicated readiness to engage in ACP conversations within six months. 
Conclusions: If HOMR can identify inpatients with elevated mortality risk on admission and trigger appropriate interventions to address unmet palliative needs, it has tremendous potential to improve end-of-life care for the frail and elderly.

\section{Identifying Older Patients at High Risk of Poor Outcomes After Major Elective Surgery}

E. Hladkowicz ${ }^{1}$, M. Taljaard ${ }^{2,3}$, G. Bryson,, ${ }^{4,5}$, P. E. Beaulé6, S. Gagné ${ }^{4,5}$, G. Hamilton ${ }^{4}$, A. Huang2, ${ }^{7}$, J. A. Joanisse ${ }^{8}$, L. T. Lavallée $^{2,9}$, D. MacDonald ${ }^{10}$, H. Moloo ${ }^{2,11}$, K. Thavorn ${ }^{2,3}$, C. van Walraven ${ }^{2,12}$, H. Yang ${ }^{13}$, A. J. Forster ${ }^{2,12}$, D. I. McIsaac ${ }^{1,2,4}$. ${ }^{1}$ Department of Anesthesiology and Pain Medicine, The Ottawa Hospital Research Institute, Ottawa, ON, Canada; ${ }^{2} \mathrm{Clinical}$ Epidemiology Program, The Ottawa Hospital Research Institute, Ottawa, ON, Canada; ${ }^{3}$ School of Epidemiology, Population Health and Preventative Medicine, University of Ottawa, Ottawa, ON, Canada; ${ }^{4}$ Department of Anesthesiology and Pain Medicine, University of Ottawa, Ottawa, ON, Canada; ${ }^{5}$ Department of Anesthesiology and Pain Medicine, The Ottawa Hospital, Ottawa, ON, Canada; ${ }^{6}$ Department of Surgery, Division of Orthopedic Surgery, University of Ottawa, Ottawa, ON, Canada; ${ }^{7}$ Department of Medicine, Division of Geriatric Medicine, The Ottawa Hospital, University of Ottawa, Ottawa, ON, Canada; ${ }^{8}$ Department of Family Medicine, Hôpital Montfort, Ottawa, ON, Canada; ${ }^{9}$ Department of Surgery, Division of Urology, The Ottawa Hospital, University of Ottawa, Ottawa, ON, Canada; ${ }^{10}$ Department of Anesthesia, Pain Management, and Perioperative Medicine, Dalhousie University, Halifax, NS, Canada; ${ }^{11}$ Department of Surgery, Division of General Surgery, The Ottawa Hospital, University of Ottawa, Ottawa, ON, Canada; ${ }^{12}$ Department of Medicine, The Ottawa Hospital, University of Ottawa, Ottawa, ON, Canada; ${ }^{13}$ Department of Anesthesia \& Perioperative Medicine, Schulich School of Medicine, London, ON, Canada.

Background: Frailty predicts adverse postoperative outcomes, yet its association with patient-reported outcomes and comparisons between preoperative frailty instruments are poorly described. We compared the accuracy, feasibility, and acceptability of the modified Fried Index [mFI] and the Clinical Frailty Scale [CFS].

Methods: Prospective multicenter cohort study. We determined preoperative $\mathrm{mFI}$ and CFS scores in 702 elective noncardiac surgery patients $>65$ years. Outcomes included death or patient-reported new disability at 90 days after surgery (primary); safety incidents, length of stay, and institutional discharge (secondary); acceptability, feasibility (tertiary).

Results: 645 participants had complete follow up. The CFS identified 297 (42.3\%) with frailty, the mFI 257 (36.6\%); 72 (11.1\%) died or experienced a new disability. Frailty was sig- nificantly associated with the primary outcome (CFS adj-OR $2.28,95 \%$ CI $1.35-1.85, p=0.002$; mFI adj-OR $2.39,95 \% \mathrm{CI}$ 1.44-3.97, $p=0.001$ ). True positive and false positive rates were not significantly different between instruments. Frailty was the only significant predictor of death or new disability in multivariable analysis. Secondary outcomes were significantly associated with frailty.

Conclusions: Older people with frailty are significantly more likely to die or experience a new patient-reported disability after surgery. The CFS was easier, more time-efficient and had less missing data.

Understanding the Role of the Public in Reducing LowValue Care: A Scoping Review

E. E. Sypes ${ }^{1}$, C. de Grood ${ }^{1}$, J. Parsons Leigh ${ }^{2}$, F. M. Clement ${ }^{1}$, H. T. Stelfox ${ }^{1,2}$, D. J. Niven ${ }^{1,2}$. ${ }^{1}$ Department of Community Health Sciences, Cumming School of Medicine University of Calgary, Calgary, AB, Canada; ${ }^{2}$ Department of Critical Care Medicine, Cumming School of Medicine, University of Calgary, Calgary, AB, Canada.

Background: Medical tests and treatments that provide no benefit to patients are considered 'low-value'. Older adults living with frailty are especially susceptible to complications from such care. It is unclear how patients and the public may be involved in initiatives to reduce low-value care and whether frailty influences the nature of this involvement.

Methods: Scoping review methodology will be employed. We will systematically search MEDLINE, Embase, and CINAHL from inception to June 2018 and grey literature using the CADTH search tool. Citations will be included if they describe patients' and/or the public's perception of and/ or involvement in reducing low-value care.

Results: This study is at the title/abstract screening stage. Included citations will be mapped onto a framework for conceptualizing the steps involved in reducing low-value care. We will examine whether frailty influences patient and public involvement in reducing low-value care, and how it might differ for patients who are not frail.

Conclusions: This study will systematically examine literature describing the involvement of patients and the public in reducing low-value care, and whether the involvement is influenced by frailty.

Training Practitioners in the Use of the CSNAT Approach-Adapted for Canadian Context 
A. M. Bitschy ${ }^{1}$, E. Donald ${ }^{2}$, G. Ewing ${ }^{3}$, G. Grande ${ }^{4}$, R. Sawatzky ${ }^{5}$, K. I. Stajduhar ${ }^{6} .{ }^{1}$ Institute on Aging \& Lifelong Health, University of Victoria, Victoria, BC, Canada; ${ }^{2}$ School of Nursing, University of Victoria, Victoria, BC, Canada; ${ }^{3}$ Centre for Family Research, University of Cambridge, Cambridge, United Kingdom; ${ }^{4}$ Division of Nursing, Midwifery, and Social Work, University of Manchester, Manchester, United Kingdom; ${ }^{5}$ School of Nursing, Trinity Western University, Langley, BC, Canada; ${ }^{6}$ Institute on Aging and Lifelong Health/School of Nursing, University of Victoria, Victoria, $\mathrm{BC}$, Canada.

Background: Family caregiving entails consider cost for Family Caregivers (FCGs) including emotional, social, financial, and physical, as well as increased mortality. We have been working with our UK colleagues to support, and make visible, the needs of FCGs within the palliative home care context. This work has resulted in the creation of the Carer Support Needs Assessment Tool (CSNAT) Approach. CFN funding allowed us to bring an effectiveness study of this work to Canada. In collaboration with Drs. Gail Ewing, and Gunn Grande, we have developed a Canadian Training Package for Practitioners.

Methods \& Results: We conducted a RCT study to determine the effectiveness of The CSNAT Approach in 9 home care offices on Vancouver Island. In addition, qualitative interviews, focus groups, and audio clips were collected about the use of The CSNAT Approach in practice. Combining this qualitative component with that of our UK colleagues we have created a training package for a Canadian audience, using context-specific language and framed within Canadian policy.

Conclusion: The CSNAT Approach Training consists of; Learning Unit 1: A practitioner's guide, and Learning Unit 2: An implementation toolkit for organizations. Currently this work is available upon request.

\section{The eDosette: Improving Medication Adherence and Reduce Regime Complexity in Community Dwelling Seniors}

F. Parascandalo ${ }^{1}$, H. Yu-Hin $\mathrm{Siu}^{2}$, B. Delleman ${ }^{2}$, J. Langevin ${ }^{2}$, D. Mangin ${ }^{2}$, M. Howard ${ }^{2}$, Q. Fang 3 , D. Price ${ }^{2}$, D. Chan ${ }^{2}$. ${ }^{1}$ David Braley Health Science Centre, McMaster University, Hamilton, ON, Canada; ${ }^{2}$ Department of Family Medicine, McMaster University, Hamilton, ON, Canada; ${ }^{3}$ Department of Engineering Physics, McMaster University, Hamilton, ON, Canada.

Background: Medication non-adherence can lead to morbidity and mortality, particularly in frail populations. This study aims to demonstrate that the eDosette intervention can report medication adherence rates and prompt medication discussions in primary care.

Methods: A multi-centre four-week prospective single arm open-label trial of the eDosette intervention with $56 \mathrm{com}-$ munity-dwelling older adults from four primary care sites. Inclusion criteria: age $65+$, taking $\geq 5$ medications (including supplements), use of blister pack/dosette (BP/D), managing medications independently, living independently, English speaking. During the intervention, information was collected on medication regimen complexity, health literacy, frailty, empowerment, medication adherence, patient satisfaction. The eDosette took images of the BP/D every half-hour, a corresponding report was created and sent for pharmacist review weekly. Thematic content of clinical encounters, change in Medication Regimen Complexity Index, and feedback on impact and areas for improvement was also collected.

Results: Mean observed adherence rate was $82 \%$ (range 49-100\%). The eDosette captured 505 missed and 2,105 late doses. 118 clinical decisions occurred; 34\% referenced an increased knowledge of medication usage or documented a medication change.

Conclusion: The eDosette intervention could play a role in medication adherence by prompting therapeutic discussions with frail older adults.

\section{How Robust are Frailty Assessments? A Systematic Review of the Application of Frailty in the Surgical Population}

H. Ting Wang ${ }^{1}$, Q. D. Nguyen 2 , C. A. Menard ${ }^{3}$, A. Morinville $^{4}$, J. P. Hirdes ${ }^{5}$, P. Hebert ${ }^{2,4}$. ${ }^{1}$ Centre Intégré Universitaire de Santé et Services Sociaux (CIUSSS) de l'est de l'île de Montréal, Hôpital Maisonneuve-Rosemont, Montréal, QC, Canada; ${ }^{2}$ Département de Médecine, Université de Montréal et Centre hospitalier de l'Université de Montréal, Montréal, QC, Canada; ${ }^{3}$ Centre hospitalier Universitaire de l'Université de Montréal, Montréal, QC, Canada; ${ }^{4}$ Centre de Recherche du Centre Hospitalier de l'Université de Montréal, Montréal, QC, Canada; 5School of Public Health and Health Systems, University of Waterloo, Waterloo, ON, Canada.

Background: Frailty is a predictor of adverse outcomes following major surgical procedures in the elderly population. Unfortunately, it is unclear how frailty screening could be practically implemented in the pre-surgical context.

Methods: We performed a systematic search of EMBASE, MEDLINE and Cochrane from inception to present time for the concept of frailty and surgery. We included interventional trials and observational cohort studies. 
Results: We found a total of 6,979 titles and 118 studies were included in our final analysis, representing a total of 2,500,040 surgical patients. There were no interventional trials. The most frequently-used instrument was the modified Frailty Index from the NSQIP dataset (24\%) followed by the Fried frailty phenotype (17\%). Frailty was most prevalent in GI oncologic surgery $(97 \%)$ and lowest gynecologic surgery $(0.6 \%)$. It also varied between $18 \%$ with NSQIP dataset and $41 \%$ with the Rockwood model. The majority of studies did not specify the timing of frailty assessments $(75 \%)$ and $26 \%$ of assessments did not report criteria used to determine frailty.

Conclusions: Important heterogeneity exists with respect to frailty implementation in the surgical setting. Future research needs to consider these elements and move towards clinical implementation and intervention.

Feeling Heard and Understood Within a Hospital Setting: Assessing Patient and Family Member's Perception of Communication Before and After a Serious Illness Conversation

J. Singh ${ }^{1}$, M. Swinton ${ }^{1}$, J. You ${ }^{2} .{ }^{1}$ Department of Health Research, Evidence and Impact, McMaster University; ${ }^{2}$ Department of Medicine, McMaster University, Hamilton, ON, Canada.

Background: Patient-centred communication is important to improving the quality of care for seriously ill, frail hospitalized patients because it promotes their values in the decision-making process. Within a hospital, serious illness conversations (SIC) promote structured communication regarding advance care planning that is both patient-centred and goal-consistent. The Heard and Understood question is one validated, self-reported quality indicator measuring the effectiveness of a SIC.

Methods: Before-after study of 8 seriously ill patients with a high degree of frailty admitted to the Medicine Ward of the Hamilton General Hospital and 13 family members. Subjects were asked before and after the SIC: "Over the past two days, how much have you felt heard and understood by doctors, nurses and hospital staff?"

Results: Prior to the SIC, $43 \%$ of patients and family members reported feeling heard and understood quite a bit or completely. Post SIC, $76 \%$ of patients and family members reported feeling heard and understood quite a bit or completely resulting in a significant increase $(p=0.004)$.

Conclusion: SIC was associated with improvement in the perception of being heard and understood by the health-care team for seriously ill, frail hospitalized patients and family members.
More-2-Eat Phase 2: Developing a Self-Serve Registry to Track Nutrition Care in Hospitals

J. M. Morrison 1 , C. V. Laur ${ }^{2}$, M. Ebad ${ }^{2}$, J. A. Dubin', ${ }^{2,3}$ H. Chen $^{2}$, L.J. Curtis ${ }^{4}$, J. J. Bell ${ }^{5,6}$, L. M. Gramlich ${ }^{7}$, H. H. Keller ${ }^{1,8} .{ }^{1}$ Department of Kinesiology, University of Waterloo, Waterloo, ON, Canada; ${ }^{2}$ School of Public Health and Health Systems, University of Waterloo, Waterloo, ON, Canada; ${ }^{3}$ Department of Statistics and Actuarial Science, University of Waterloo, Waterloo, ON, Canada; ${ }^{4}$ Department of Economics, University of Waterloo, Waterloo, ON, Canada; ${ }^{5}$ School of Human Movement and Nutrition Sciences, The University of Queensland, Brisbane, Australia; ${ }^{6}$ The Prince Charles Hospital, Queensland, Australia; ${ }^{7}$ Faculty of Medicine and Dentistry, University of Alberta, AB, Canada; ${ }^{8}$ Schlegel-UW Research Institute for Aging, Waterloo, ON, Canada.

Background: More-2-Eat (M2E) aims to minimize malnutrition and associated frailty in hospitals by systematizing nutrition care using the Integrated Nutrition Pathway for Acute Care (INPAC). Initiation of INPAC in 5 hospitals (Phase 1) demonstrated audit \& feedback was key to behavior change among participants (i.e., hospital personnel, typically dietitians). This study describes the development of a registry to allow hospitals to self-audit INPAC implementation (Phase 2).

Methods: M2E researchers designed the registry with input from Phase 1 participants (Jan-May 2018); all participants tested the system (June 2018). The audit questions verified key patient-tailored nutrition care practices. REDCap, a secure online platform, was used for data entry and a macro-enabled report template was generated using Microsoft Excel.

Results: Several challenges were overcome (e.g., firewalls) and lessons learned considering the end user (i.e., hospital personnel) to develop a user-friendly self-serve registry. Users generate automatic reports (e.g., tables/graphs) by entering INPAC audit data into REDCap, and loading the downloadable data file into the report template. Instructions and a video are provided.

Conclusions: A self-serve registry that supports audit \& feedback behaviour change strategies will improve sustainability of the M2E model to address nutrition care in hospitals.

\section{Diarrhea During Mechanical Ventilation: A Multicenter Study}

J. Dionne ${ }^{1}$, E. Duan ${ }^{1}$, F. Clarke 2 , L. Hand ${ }^{3}$, T. Millen ${ }^{4}$, G. Sandu $^{5}$, J. Hodder ${ }^{5}$, M. Santos 5 , S. Shah ${ }^{6}$, M. Trembley ${ }^{7}$, B. Gomes $^{8}$, L. Leclair ${ }^{8}$, K. Montroy ${ }^{8}$, I. Watpool ${ }^{9}$, R. Porteous ${ }^{9}$, S. Acres ${ }^{9}$, D. Foster ${ }^{10}$, F. Auld ${ }^{11}$, V. Williams ${ }^{12}$, J. Marchand ${ }^{13}$, 
J. Campisi ${ }^{14}$, N. Alam ${ }^{15}$, M. Lebrassier ${ }^{16}$, P. Thompson ${ }^{17}$, T. Hewer $^{17}$, D. Gilles ${ }^{18}$, M. Hunt ${ }^{19}$, I. Georgescu ${ }^{19}$, T. Boyd ${ }^{19}$, J. Lys ${ }^{20}$, N. Marten ${ }^{20}$, E. Campbell ${ }^{21}$, T. Bentall ${ }^{22}$, K. Kavikondala $^{23}$, S. Willems ${ }^{24}$, Z. Panchbhaya ${ }^{25}$, J. Booth ${ }^{26}$, S. Ruddell $^{26}$, B. Richter ${ }^{27}$, D. Tassy ${ }^{28}$, R. Jesso ${ }^{29}$, N. Marinoff ${ }^{30}$,

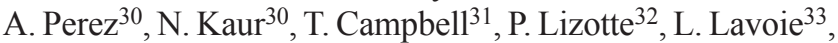
M. Dionne ${ }^{33}$, L. Saunders ${ }^{3}$, N. Zytaruk ${ }^{34}$, D. Heels-Ansdell ${ }^{3}$, J. Johnstone ${ }^{34}$, D. Cook ${ }^{3}$. ${ }^{1}$ Department of Medicine, McMaster University, Hamilton, ON, Canada; ${ }^{2}$ St. Joseph's Healthcare, Hamilton, ON, Canada; ${ }^{3} \mathrm{McMaster}$ University, Hamilton,

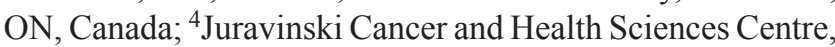
Hamilton, ON, Canada; ${ }^{5}$ St. Michael's Hospital, Toronto, ON, Canada; ${ }^{6}$ Mount Sinai Hospital, Toronto, ON, Canada; ${ }^{7}$ Hôpital de 1'Enfant Jésus, Québec City, QC, Canada; ${ }^{8}$ Ottawa Civic Hospital, Ottawa, ON, Canada; ${ }^{9}$ Ottawa General Hospital, Ottawa, ON, Canada; ${ }^{10}$ Vancouver General Hospital, Vancouver, BC, Canada; ${ }^{11}$ Royal Jubilee Hospital, Victoria, BC, Canada; ${ }^{12}$ Hôpital du Sacré Coeur de Montréal, Montreal, QC, Canada; ${ }^{13}$ Université de Sherbrooke; Sherbrooke, QC, Canada; ${ }^{14}$ Royal Victoria Hospital, Montreal, QC, Canada; ${ }^{15}$ Montreal General, Montreal, QC, Canada; ${ }^{16} \mathrm{CHUM} \mathrm{Re}-$ search Center, Montreal, QC; ${ }^{17}$ Royal Alexandra Hospital, Edmonton, AB, Canada; ${ }^{18}$ Queen Elizabeth Health Sciences Center, Halifax, NS, Canada; ${ }^{19}$ Kingston General Hospital, Kingston, ON, Canada; ${ }^{20}$ St. Boniface Hospital, Winnipeg, MB, Canada; ${ }^{21}$ London Health Sciences Centre, Victoria Site, London, ON, Canada; ${ }^{22}$ London Health Sciences University Hospital, London, ON, Canada; ${ }^{23}$ St. Joseph's Health Centre, Toronto, ON, Canada; ${ }^{24}$ Royal Columbian Hospital, New Westminster, BC, Canada; ${ }^{25}$ Brampton Civic Hospital, Brampton, ON, Canada; ${ }^{26}$ Foothills Medical Centre, Calgary, AB, Canada; ${ }^{27}$ Niagra Health, St Catharines, ON, Canada; ${ }^{28}$ Hôpital Maisonneuve-Rosemont, Montreal, QC, Canada; ${ }^{29}$ Grand River Hospital, Kitchener, ON, Canada; ${ }^{30}$ Sunnybrook Health Sciences Centre, Toronto, ON, Canada;

${ }^{31}$ Joseph Brant Hospital, Burlington, ON, Canada; ${ }^{32}$ Institut Universitaire de Cardiologie et de Pneumologie de Québec, Québec, QC, Canada; ${ }^{33}$ Centre de Recherche de l'Hôtel-Dieu de Lévis, Sainte-Marie, QC, Canada; ${ }^{34}$ University of Toronto, Toronto, ON, Canada.

Background: It remains unclear what factors predispose to diarrhea when older patients (age $>65$ ) become critically ill. Our objective was to describe the incidence of, and risk factors for, diarrhea nested within a probiotics trial (PROSPECT).

Methods: A nested cohort of diarrhea in older adults within a trial testing Lactobacillus rhamnosus $G G$ was performed. Bowel movements [BMs] frequency, and Bristol Stool Type were documented. Daily data was collected on risk factors for diarrhea.

Results: Among 1,243 invasively mechanically-ventilated patients the incidence of diarrhea was $59.5 \%$ (defined as $\geq 3$ Bristol Type 6/7 BMs any day). Regression analysis showed that age and illness severity didn't predispose to diarrhea. However, frailty score $\geq 5$ (Hazard Ratio [HR] 1.21, 95\% CI 1.04-1.42) and gastrointestinal disease (inflammatory bowel disease, irritable bowel syndrome or celiac disease) (HR $1.77,95 \%$ CI 1.21-2.60) significantly increased the risk. (HR 1.70, 95\%CI 1.38-2.09), proton-pump-inhibitors (HR 1.29, 95\%CI 1.06-1.57), nutrition (HR 1.35, 95\%CI 1.01-1.79), laxatives (HR 2.11, 95\%CI 1.75-2.53), and Clostridium difficile infection (HR 3.48, 95\%CI 1.75-6.93) significantly increased diarrhea risk.

Conclusion: Diarrhea affects most mechanically-ventilated patients, particularly frail patients. Recognizing frail patients experiencing diarrhea emphasizes the importance of intervening on modifiable risk factors to reduce the impact on these patients.

\section{The Association Between Physician Intensity of Episodic Antibiotic Prescribing and the Prescribing of Opioids, Benzodiazepines and Proton Pump Inhibitors: A Window for Improving Physician Prescribing Behavior to Frail Adults}

K. L. Quinn ${ }^{1,2,3,4}$, M. A. Campitelli ${ }^{2}$, C. Diong ${ }^{2}$, N. Dane$\operatorname{man}^{2,6}$, N. Stall ${ }^{1,2,3,4,5}$, A. M. Morris ${ }^{1,4}$, A. S. Detsky ${ }^{1,3,4}$, L. Jeffs $^{7}$, C. J. Maxwell ${ }^{8}$, C. M. Bell ${ }^{1,2,3,4}$, S. E. Bronskill ${ }^{2,3,5,6}$. ${ }^{1}$ Department of Medicine, University of Toronto, Toronto, ON, Canada; ${ }^{2}$ Institute for Clinical Evaluative Sciences, Toronto, ON, Canada; ${ }^{3}$ Institute of Health Policy, Management and Evaluation, University of Toronto, Toronto, ON, Canada; ${ }^{4}$ Department of Medicine, Sinai Health System, Toronto, ON, Canada; ${ }^{5}$ Women's College Research Institute, Women's College Hospital, Toronto, ON, Canada; ${ }^{6}$ Sunnybrook Research Institute, Toronto, ON, Canada; ${ }^{7} \mathrm{Li}$ Ka Shing Knowledge Institute, St. Michael's Hospital, Toronto, ON, Canada; ${ }^{8} \mathrm{Sch}$ ool of Public Health, University of Waterloo, Waterloo, ON, Canada.

Background: Identifying the individual physician responsible for prescribing chronic medications is challenging since multiple physicians are often involved. The extent to which a physician's episodic antibiotic prescribing practices are associated with the rate of prescription of other chronically prescribed medications has not been established. Demonstration of this association may help focus rational prescribing efforts for multiple medications simultaneously, especially in vulnerable frail adults.

Methods: This cross-sectional study used linked health administrative data to examine physician prescribing practices in long-term care facilities in Ontario during 2015. Physicians were assigned all residents to whom they prescribed at least one medication. The main exposure was physicianlevel intensity of antibiotic prescribing to their assigned 
residents. The primary outcome was the prescription of an opioid, benzodiazepine or proton-pump inhibitor. The association between physician antibiotic prescribing intensity and prescribing of these medications was determined using hierarchical logistic regression.

Results: Compared with average-intensity antibiotic prescribers, high-intensity prescribers had an increased risk of prescribing a benzodiazepine (OR 1.14 [95\% CI, 1.05-1.25]); opioid (OR 1.19 [95\% CI, 1.10-1.30]); or proton pump inhibitor 1.27 [95\% CI, 1.17-1.38], $p<0.001$ ). Frail individuals were also significantly more likely to receive one of these medications.

Conclusions: The intensity of a physician's antibiotic prescribing was significantly associated with the prescription of a potentially high-risk medication. Episodic prescribing practices may identify physicians for targeted intervention to optimize their use of medications, especially in frail individuals.

\section{The Role of Body Composition in Predicting Outcomes in the Elderly Following Acute Abdominal Surgery}

M. Alghamdi, V. Baracos, C. Karvellas, T. Churchill, R. G. Khadaroo. Department of Surgery, University of Alberta, Edmonton, AB, Canada.

Background: As the elderly population is increasing, their need for emergency surgery is expected to rise. With aging, there is a decrease in skeletal muscle mass and an increase in visceral fat. To date, the role of age-associated changes in body composition with outcome is unclear. We aimed to examine the association of body composition identified by computed tomography (CT) scan with in-hospital mortality and postoperative complications.

Methods: A retrospective cohort of 215 patients aged $\geq 65$ years underwent acute abdominal surgery between 2008 and 2010 at the University of Alberta Hospital was analysed. CT scan at L3 was used to measure height-adjusted surface area $\left(\mathrm{cm} / \mathrm{m}^{2}\right)$ of muscularity, visceral fat, and subcutaneous fat, and, their radiodensities in Hounsfield Units (HU). Logistic regression was used to assess the relationship between body composition and in-hospital mortality and postoperative complications.

Results: Multivariate analysis identified muscularity (aOR: $0.922,95 \%$ CI: $0.863-0.985, p$-value $=0.016$ ) was a strong predictor of in-hospital mortality while subcutaneous fat radiodensity was not (aOR: 1.028, 95\% CI: 0.999-1.058, $p$-value $=0.055)$. Muscularity (aOR: $0.977,95 \%$ CI: 0.935 $1.021, p$-value $=0.307)$ and subcutaneous fat radiodensity $($ aOR: $1.013,95 \%$ CI: 0.990-1.036, $p$-value $=0.272)$ were not significantly associated with postoperative complications.
Conclusion: Muscularity was an independent predictor of in-hospital mortality. CT-identified body composition measurements can be used for risk stratification and as a potential modifiable risk factor for intervention.

\section{Implementing the 'Frailty Portal' in Community Primary Care Practice: Evaluating Feasibility, Effects and Expansion Needs}

F. Burge ${ }^{1}$, P. Moorhouse ${ }^{2}$, T. Sampalli ${ }^{3}$, L. Bedford ${ }^{3}$, L. Edwards $^{3}$, R. Gibson 3,4 , L. Mallery ${ }^{2}$, D. Taylor ${ }^{3}$, G. Warner ${ }^{5}$, A. Harnish $^{3}$, V. Law ${ }^{6}$, B. J. Lawson ${ }^{1}$, S. Wood ${ }^{3}$, M. Buckler ${ }^{2}{ }^{1}$ Department of Family Medicine, Dalhousie University, Halifax, NS, Canada; ${ }^{2}$ Department of Medicine, Nova Scotia Health Authority, Halifax, NS, Canada; ${ }^{3}$ Primary Health Care, Nova Scotia Health Authority, Halifax, NS, Canada; ${ }^{4}$ Department of Family Medicine, Nova Scotia Health Authority, Halifax, NS, Canada; ${ }^{5}$ School of Occupational Therapy, Dalhousie University, Halifax, NS, Canada; ${ }^{6}$ Memorial University, St. John's, NL, Canada.

Background: Routine frailty identification is not standard practice in primary care in Nova Scotia. The Nova Scotia Health Authority, Central Zone, developed a web-based 'Frailty Portal' to assist primary care providers with frailty identification and care planning.

Methods: A mixed methods prospective study was completed to understand Portal feasibility and scalability in primary health care. Participants included family physicians, nurse practitioners, frail patients, caregivers, and other stakeholders.

Results: Providers reported desire to improve care for frail patients. They were more confident in their ability to assess, discuss, and offer frailty care options. Technical issues, follow through with care planning and time constraints were identified as challenges. Lack of practice resources for change, follow-up, reimbursement, training opportunities and integration with existing electronic medical records were barriers for use.

Conclusions: Frailty Portal implementation into community primary care is very complex. The current organization of primary care practice requires changes management to successfully integrate the Frailty Portal into practice. Once in use, the portal shows promise for supporting primary care physicians in providing complex care for frail patients and their caregivers in the community.

Setting the Stage for a Co-design Process: A Scoping Study of mHealth Tools to Support Care Coordination 
P. Fernandes, J. Elliott, P. Stolee. School of Public Health and Health Systems, University of Waterloo, Waterloo, ON, Canada.

Background \& Objectives: Older adults living with frailty may experience numerous chronic conditions and see multiple health-care providers, impacting their care coordination practices (Lorig et al., 1999). Health information technologies can support care planning and communication between patients and providers. This study aimed to: i) review the literature for mHealth tools supporting care coordination; and ii) use these data to inform a consultation process with older adults and providers.

Methods: This scoping study used the methodology of Arksey \& O'Malley (2005), as supplemented by Levac and colleagues (2010). The consultation included three focus group interviews ( $\mathrm{n}=11$ older adults; $\mathrm{n}=6$ caregivers) and five individual interviews ( $\mathrm{n}=4$ providers; $\mathrm{n}=1$ caregiver). Interviews were audio-recorded, transcribed verbatim, and analyzed using thematic analysis (Braun \& Clarke, 2006), supported by NVivo 11 .

Results: The literature identified features, strengths and implementation issues of current tools. The consultation prioritized features and resources important for supporting older adults living with frailty and their current care coordination practices.

Conclusions: This study confirmed that older adults and providers have an interest in using mHealth, and highlighted the value of engaging older adults and providers in tool development. These data will inform the development of an mHealth tool to support care coordination.

\section{Paradoxical Relationship of Muscle Fibre Cross-Sectional Area in Older Emergency Surgery Patients}

G. Ali ${ }^{1}$, A. Dunichand-Hoedl ${ }^{2}$, S. Y. Salim³ , V. C. Mazurak ${ }^{2}$, V. E. Baracos ${ }^{1}$, R. G. Khadaroo ${ }^{3} .{ }^{1}$ Department of Oncology, Faculty of Medicine and Dentistry, University of Alberta, Edmonton, AB, Canada; ${ }^{2}$ Department of Agricultural, Food $\&$ Nutritional Science, Faculty of Agriculture Life and Environmental Sciences, University of Alberta, Edmonton, AB, Canada; ${ }^{3}$ Department of Surgery \& Division of Critical Care Medicine, Faculty of Medicine and Dentistry, University of Alberta, Edmonton, AB, Canada.

Background: The number and cross-sectional area (CSA) of individual fibers within a muscle determine the muscle's capacity to generate force, and both lessen with age. Muscle fibre area is normally positively associated with strength. A variety of indices of frailty and function are available for older patients, however the corresponding muscle morphology is limited.
Methods: Rectus abdominis (RA) muscle biopsies were collected during surgery from $n=24$ female patients 65-94 yr of age undergoing emergency surgery. Frozen muscle samples were mounted in cross section and immunostained for muscle fiber area $\left(\mu \mathrm{m}^{2}\right)$. Muscle cross sectional area $\left(\mathrm{CSA} ; \mathrm{cm}^{2}\right)$ was calculated from CT images at lumbar 3 obtained from medical records of patients. Correlations were determined using IBM SPSS software.

Results: Mean RA muscle fibre cross-sectional area was 3437 $\mu \mathrm{m}^{2}$ (range 1452-6705 $\mu \mathrm{m}^{2}$ ), however this was not correlated with age $(p=0.928)$, BMI $(p=0.109)$ or frailty $(p=0.540)$. Patients with larger muscle fibers performed more poorly in sit-to-stand tests $(p=0.030)$ and had increased intramuscular adipose tissue $(p=0.036)$.

Conclusions: Larger muscle fibre area is associated significantly with lower sit-to-stand performance. Further research is required to understand why larger muscle fibres in older adults are not associated with better strength and function.

\section{Pre-morbid Characteristics of Home Care Clients Predict Delayed Discharge in Acute Hospitals: Results from a Retrospective Cohort Study}

J. P. Hirdes ${ }^{1}$, G. A. Heckman ${ }^{1}$, P. C. Hebert ${ }^{2}$, A. Morinville ${ }^{2}$, A. P. Costa ${ }^{3}$, S. A. Arthur ${ }^{1} .{ }^{1}$ School of Public Health and Health Systems, University of Waterloo, Waterloo, ON, Canada; ${ }^{2}$ Université de Montréal Hospital Research Centre, Montreal, QC, Canada; ${ }^{3}$ Department of Clinical Epidemiology \& Biostatistics, McMaster University, Hamilton, ON, Canada.

Background: Better identification of frail persons with complex needs early during a hospitalization may prevent iatrogenic complications, reduce length of stay and increase the likelihood of a successful discharge home.

Methods: In this retrospective cohort study, we linked home care assessment records based on the Resident Assessment Instrument for Home Care (RAI-HC) of 210,931 hospitalized patients with their Discharge Abstract Database records. Following descriptive statistics, we then undertook multivariable logistic regression analyses to identify predictive factors for delayed discharge in hospital.

Results: Characteristics that were predictive of delayed discharge included advanced age (OR: $2.71,95 \%$ CI 2.55 2.89 ), social vulnerability (OR: $1.27,95 \%$ CI $1.08-1.49$ ), Alzheimer's disease and related dementias (OR: 1.27, 95\% CI 1.23-1.3), and problematic behaviours such as wandering (OR: $1.29,95 \%$ CI 1.22- 1.38).

Conclusion: This study demonstrates the potential utility of home care assessments for predicting delayed discharge after 
a hospital admission which could lead to improved disposition planning by proactively identifying patients most at-risk for adverse outcomes. Implementing a common approach and language throughout the system may assist in guiding early interventions at hospital admission.

The NORC Index: A Tool to Identify and Prioritize Naturally-Occurring Retirement Communities

A. Jones ${ }^{1}$, L. Salam-White ${ }^{2}$, D. Tanner ${ }^{2}$. 1Department of Health Research Methods, Evidence and Impact, McMaster University, Hamilton, ON, Canada; 2Hamilton-Niagara-Haldimand-Brant Local Health Integration Network, ON, Canada.

Background: Naturally-occurring retirement communities (NORCs) are concentrations of older adults in locations not designed to support the needs of a large number of independently-living seniors. The identification and support of NORCs has become an integral part of aging strategies, including in Ontario.

Methods: We used administrative health and census data to create a NORC index to identify and prioritize potential NORCs. Four measures were included in the index: density of seniors, marginalization, volume of home care referrals, and rate of emergency department (ED) visits among seniors. Each measure was calculated for postal codes in the HNHB region of Ontario using census population counts, the Ontario Marginalization Index, and administrative home care and ED records. The measures were converted into percentiles and summed to generate the NORC index (0-400).

Results: The NORC index was calculated for 39,522 postal codes in HNHB with values ranging from 395 to 62 . Consultations with regional health planners and screening of top scoring sites indicated that the index has content and construct validity.

Conclusions: We created the NORC index to help identify and prioritize NORCs for support. The index is based on administrative health and census data mapped to postal codes and demonstrates content and construct validity.

\section{A Protocol for the Fit-Joint Pilot RCT of A Multi-modal Intervention in Frail Patients with Osteoarthritis}

A. M. Negm ${ }^{1,2}$, C. C. Kennedy ${ }^{1}$, G. Ioannidis ${ }^{1}$, O. GajicVeljanoski ${ }^{1}$, J. Lee ${ }^{1,3}$, L. Thabane ${ }^{4}$, J. D. Adachi ${ }^{3}$, S. Marr ${ }^{1,3}$, A. Lau $^{3}$, S. Atkinson ${ }^{5}$, D. Petruccelli ${ }^{6}$, J. DeBeer $^{6}$, M. Winemaker $^{6}$, V. Avram ${ }^{6}$, D. Williams ${ }^{6}$, D. Armstrong 3 , B. Lumb ${ }^{3}$, A. Panju ${ }^{3}$, J. Richardson ${ }^{2}$, A. Papaioannou ${ }^{1,3} \cdot{ }^{1}$ Geriatric
Education and Research for the Aging Sciences (GERAS), St Peter's Hospital, Hamilton ON, Canada; ${ }^{2}$ School of Rehabilitation Science, McMaster University, Hamilton, ON, Canada; ${ }^{3}$ Department of Medicine, McMaster University, Hamilton ON, Canada; ${ }^{4}$ Department of Clinical Epidemiology and Biostatistics, McMaster University, Hamilton ON, Canada; ${ }^{5}$ Department of Pediatrics, McMaster University, Hamilton ON, Canada; ${ }^{6}$ Department of Surgery, Division of Orthopaedics, McMaster University, Hamilton, ON, Canada.

Background: It is important to determine whether targeting frailty in Joint replacement patients improves post-operative outcomes. Our primary objective is to examine the feasibility of a pilot RCT comparing a multi-modal frailty intervention (MMFI) to usual care in pre-frail/frail older adults undergoing hip or knee replacement. Secondary objectives include examining the effectiveness of the MMFI in 1) improving frailty and mobility using Fried Frailty Phenotype (FFP) and Short Performance Physical Battery respectively, and 2) reducing post-operative health-care services use (length of stay; rate of complications; hospital re-admissions, emergency room visits).

Methods: Participants who are: 1) $\geq 65$ years old; 2) Pre-frail (score of 1-2; FFP) or frail (score of 3-5; FFP); 3) having elective unilateral hip or knee replacement; and 4) having surgery wait times between 3-10 months will be recruited from the Regional Joint Assessment Program in Hamilton and randomized to the intervention or usual care group. The MMFI consists of: 1) Tailored exercise; 2) Protein and vitamin D supplementation; and 3) Medication review.

Conclusion: This is the first study to examine the effect of MMFI in pre-frail/frail older adults undergoing joint replacement. This study will inform the planning and designing of MMFIs in patients waiting for joint replacement.

Exploring Factors Associated with Burden of Care Among Caregivers of Cognitively-Impaired Frail Elderly People Facing Housing Decisions

A. Boucher ${ }^{1,2}$, J. Haesebaert ${ }^{1,2}$, A. Freitas ${ }^{1,2}$, R. Adekpedjou $^{1,2}$, M. Landry ${ }^{3}$, H. Bourassa ${ }^{4}$, S. Dawn ${ }^{5,6}$, J. Croteau ${ }^{1,2}$, F. Légaré ${ }^{1,2}$. ${ }^{1}$ Laval University, Québec City, QC, Canada; ${ }^{2}$ Centre de recherche sur les soins et les services de première ligne de l'Université Laval (CERSSPL-UL), Québec City, QC, Canada; ${ }^{3}$ Université du Québec à Trois-Rivières, TroisRivières, QC, Canada; ${ }^{4}$ Caregiver partner; ${ }^{5}$ Ottawa Hospital Research Institute, Ottawa, ON, Canada; ${ }^{6}$ School of Nursing, University of Ottawa, Ottawa, ON, Canada.

Background: Making health-related decisions for loved ones with cognitive impairment may contribute to caregiver burden 
of care. We explored factors associated with burden of care among caregivers of cognitively-impaired frail elderly people facing housing decisions.

Methods: Secondary data analysis from a cluster randomized trial (cRT) conducted in 16 home-care organizations. This cRT assessed the impact of shared decision making (SDM) on the role of caregivers in making housing decisions for cognitivelyimpaired frail elderly people. We assessed caregiver burden of care with the Zarit Burden Inventory Scale (ZBI). Guided by Pallett's conceptual framework, we studied the effect of caregivers' and elderly persons' characteristics, their relationship, and decision-making outcomes on burden of care using multilevel regression.

Results: Among 296 caregivers, the mean age was 62.6 years, $74.7 \%$ were women, and $59.9 \%$ were caring for a parent. The mean ZBI score was 29.8/88( $\mathrm{SD}=17.5)$. In multilevel regression, urban settings, being female, decision regret, decisional conflict, the actual housing decision made, being a spouse or parent, and being involved in the SDM process were associated with higher ZBI.

Conclusion: These results will help designing future interventions to prevent and/or reduce burden of care in caregivers facing housing decisions for cognitively impaired frail elderly relatives.

\section{Exploring the Differences Between Pre-ICU and 1-Year CFS Scores: A Follow-Up Study of ICU Survivors}

A. M. Takaoka ${ }^{1}$, F. J. Clarke ${ }^{1}$, M. S. Shears ${ }^{1}$, L. Thabane ${ }^{1}$, J. Muscedere $^{2}$, D. J. Cook ${ }^{1,3}$. ${ }^{1}$ Department of Health Research Methods, Evidence and Impact McMaster University, Hamilton, ON, Canada; ${ }^{2}$ Departments of Medicine and Critical Care, Queens University, Kingston, ON, Canada; ${ }^{3}$ Department of Medicine, McMaster University, Hamilton, ON, Canada.

Background: Frailty is associated with increased hospital length of stay, and functional dependence post-hospital discharge. The 9-point Clinical Frailty Scale (CFS) is designed to screen for frailty using chart review and patient/family member conversations. Our objective was to compare CFS values pre-ICU and at 1-year follow-up.

Methods: In a single-center prospective study, critically ill patients admitted to ICU for ${ }^{3} 24$ hours had baseline CFS scores assigned; scores were determined at 1 -year post-ICU admission through patient/family telephone interviews. Mean differences (MD) and mean change scores (MCS) were calculated; $\mathrm{CFS}^{3} 5$ was considered frail.

Results: 100 patients were enrolled, 36 of which had $12( \pm 2)$ months follow-up (26 died in hospital, 18 died post-discharge within 1-year, 19 could not be contacted, 1 declined). Of these, $42 \%$ had an increased CFS score at 1 -year, $39 \%$ were unchanged, while $19 \%$ had a decreased score. Overall, prehospital and 1 -year CFS scores were similar $(\mathrm{MD}=0.42$, $p=0.07)$. There was no difference between pre-hospital and 1 -year CFS scores in 17 frail patients (MCS $=0.71$ ) compared to 19 non-frail patients $(\mathrm{MCS}=0.16)(\mathrm{MD}=0.55, p=0.22)$.

Conclusions: Overall, CFS scores at 1-year versus pre-critical illness did not change significantly. Several methodologic challenges influence the understanding of frail ICU patients' trajectories through long-term follow-up.

\section{Sedentary Behaviour in Long-Term Care}

A. Lee ${ }^{1}$, D. R. Bouchard ${ }^{1}$, M. Sénéchal ${ }^{2} .{ }^{1}$ Faculty of Kinesiology, University of New Brunswick, Fredericton, NB, Canada; ${ }^{2}$ York Care Centre, Fredericton, NB, Canada.

Background: Not many studies have been looking at the time spent and consequences of sitting for older adults, especially by residents in long-term care (LTC). Sitting is associated with mortality risk and independence. It is currently unknown if sitting time predicts changes in mobility status.

Methods: A total of 20 residents in a LTC facility were recruited from three categories of mobility: independent $(\mathrm{N}=10)$, assistance in transfers $(\mathrm{N}=5)$, and dependent on mechanical assistance for transfers $(\mathrm{N}=5)$. An ActivPal was attached to the leg with Tegaderm tape for seven days continuously. Twelve months after, mobility categories were reviewed.

Results: The sample was 78 years old and included 12 women. The group had an average of $21.1 \pm 2.6$ hours per day spent doing sedentary activities and no significant difference was observed between assistance in transfers $(22.2 \pm 2.2)$ and dependent (22.6 \pm 1.6$) ; p=0.76)$. At follow-up evaluation, five participants decreased their mobility level. Despite a $7.5 \%$ lower baseline sedentary time in the group that has not changed mobility category, no significant difference was observed.

Conclusions: Older adults living in LTC are highly sedentary. More research is needed to evaluate if sedentary behavior predicts mobility changes.

\section{Is Duration of Exercise Associated with Greater Physical Function in Older Adults?}

A. Mayo, ${ }^{1,2}$, T. J. Hrubeniuk ${ }^{1,3}$, M. Keshavarz ${ }^{1}$, M. Sénéchal ${ }^{1,3}$, D. R. Bouchard ${ }^{1,3}$. ${ }^{1}$ Cardio-metabolic Exercise \& Lifestyle Laboratory, University of New Brunswick, Fredericton, NB, 
Canada; ${ }^{2}$ Interdisciplinary Studies, School of Graduate Studies, University of New Brunswick, Fredericton, NB, Canada; ${ }^{3}$ Faculty of Kinesiology, University of New Brunswick, Fredericton, NB, Canada.

Background: To improve physical function (PF), physical activity (PA) guidelines for older adults recommend bouts a minimum 10 minutes. Spontaneous PA $(<10$ minutes) has also shown to benefit; however, less research exists. The purpose of this study was to determine the association between various patterns of PA and the likelihood of greater PF in older adults.

Methods: Older adults (65+) from the National Health and Nutrition Examination Survey $(\mathrm{n}=1,274)$ were included. PA lasting $1,5,10$, and 30 minutes was quantified using accelerometers. PF was assessed using a Likert scale reflecting the self-reported capability to complete 11 tasks. A function score was made using factor analysis. Logistic regression analyses calculated the association between PA bout length and the likelihood of above average function.

Results: PA performed in 1-minute (odds ratio [OR]) 1.03; 95\% confidence interval [CI], 1.02-1.04), 5-minute (OR 1.03; CI 1.01-1.04), 10-minute bouts (OR 1.03; CI 1.02-1.04), and 30-minute (OR 1.04; CI 1.02-1.06) was associated with greater PF following adjustment for confounders.

Conclusion: The findings suggest that patterns of PA shorter than 10-minutes in duration are associated with greater PF in older adults.

\section{Physician Perspectives of Medical Assistance in Dying (MAiD): A Scoping Review}

C. Robertson, E. A. Read. University of New Brunswick, Fredericton, NB, Canada.

Background: Despite the legalization of medical assistance in dying (MAiD) in Canada in 2016, access to MAiD is largely dependent upon physician willingness to provide this service. This study examined the literature globally regarding physicians' perspectives towards MAiD.

Methods: A scoping review of the literature was conducted. Papers published from May 2012 to April 2018 were included. Papers that focused on other health-care providers, and any acts that would be considered illegal in Canada were excluded. This was meant to identify perspectives that would be relevant specifically to physicians in Canada.

Results: A total of 149 papers were identified, of which 74 were included in the final analysis. Of the 74 papers included, 24 originated from Canada, with most of those being opinion pieces. Early thematic analysis points to the importance of religion, the Hippocratic oath, patient dignity and ending suffering, as factors in shaping the physician perspective towards MAiD.

Conclusions: Legalization of MAiD is an important first step towards providing terminally ill Canadians with control over the conditions of their death. There is a potential that physician perspectives on MAiD can influence access for patients, but further research is needed in the Canadian context.

\section{Preoperative Cardiac Surgery Frailty Assessment: Alberta Provincial Prospective Cohort Results}

C. L. Montgomery ${ }^{1,2,8}$, C M. Norris ${ }^{4,5,7}$, D. B. Rolfson ${ }^{2,3}$, S. R. Meyer ${ }^{4}$, H. T. Stelfox ${ }^{6,7}$, M. Zibdawi ${ }^{1}$, S. M. Bagshaw ${ }^{1,2,7}$. ${ }^{1}$ Department of Critical Care Medicine, University of Alberta, Edmonton, AB, Canada; ${ }^{2}$ Department of Medicine, University of Alberta, Edmonton, AB, Canada; ${ }^{3}$ Division of Geriatric Medicine, University of Alberta, Edmonton, AB, Canada; ${ }^{4}$ Division of Cardiac Surgery, Faculty of Medicine \& Dentistry, University of Alberta, Edmonton, AB, Canada; ${ }^{5}$ Faculty of Nursing, University of Alberta, Edmonton, AB, Canada; ${ }^{6}$ Cumming School of Medicine, University of Calgary, Calgary, $\mathrm{AB}$, Canada; ${ }^{7}$ Alberta Health Services, Edmonton, AB, Canada $;{ }^{8}$ Canadian Frailty Network Interdisciplinary Fellow, Canadian Frailty Network, Kingston, ON, Canada.

Background: It is imperative to identify patients whose preoperative frailty puts them at risk when undergoing complex and invasive procedures. Frailty screening may aid in the design of novel interventions to optimize outcomes in cardiac surgery patients.

Methods: A prospective population-based cohort of Alberta patients aged $\geq 50$ years referred for cardiac surgery were screened for frailty using the Clinical Frailty Scale (CFS). Patients were followed postoperatively to assess vital status, health services use and health-related quality-of-life (HRQL) (EQ-VAS).

Results: Results indicated the overall $(\mathrm{n}=529)$ median (IQR) age was 67 (14) years, $26 \%$ were female, and $10 \%$ were screened as frail (CFS $\geq 5)$ preoperatively. Parsonnet (21 vs. 12 ) and EuroSCORE (8 vs. 5) were higher in frail compared to nonfrail ( $p<0.001$ for each). The median ICU stay (63 vs. 29 hours) and postoperative hospitalization (10 vs. 7 days) were longer for frail patients ( $p<0.001$ for each). Frail patients reported lower HRQL preoperatively, at 6 and 12 months on the EQ-VAS $(p<0.001)$.

Conclusions: Although relatively uncommon, frailty was associated with greater perioperative morbidity and health resource 
use. Interventions that incorporate frailty into preoperative risk measurement may optimize cardiac surgery outcomes.

\section{Frail older People with Fragmented Rural Care}

H. D. Marshall. Department of Geography and Planning, Queen's University, Kingston, ON, Canada.

Background: The provision of Primary Health Care (PHC) to frail older adults in rural Canada is spatially inequitable in comparison to those same adults in urban settings (Rosenberg $\&$ Moore, 1997). I will demonstrate that improvements in health and social outcomes for the community-dwelling frail elderly in rural small towns are possible through the adoption of evidence-based practices in integrated interdisciplinary PHC delivery.

Methods: A sequential explanatory, four-phase, mixedmethods design will be used. Phases $1 \& 2$ seek to understand the quantitative relationships between rurality and frailty and then between the levels of PHC integration, and frailty. Phases $3 \& 4$ will analyze one highly-integrated service rich community and one less-integrated service-poor community in a qualitative comparative case study.

Potential Impact: Improved health and social outcomes for frail older adults living in rural settings are possible through improvements in interprofessional collaborative practice and the adoption of interdisciplinary tools for increasing integration.

Next Steps: Building on prior international research in integrated practice, this work will extend those interventions to rural small towns in Canada where urban-rural inequity has led to fragmentation - a pressing problem.

Exploring the Needs and Associated Out-of-Pocket Expenses of Older People Living with Frailty who Wish to Age in Place

E. M. Moody ${ }^{1,2}$, R. Martin-Misener ${ }^{1}$, G. Warner ${ }^{2} .{ }^{1}$ School of Nursing, Dalhousie University, Halifax, NS, Canada; ${ }^{2}$ School of Occupational Therapy, Dalhousie University, Halifax, NS, Canada.

Background: There has been increasing recognition of the need to support older people living with frailty in the community. A range of supports are needed to enable this population to live well at home and are often associated with out-of-pocket expenses for individuals or caregivers. Financial considerations are important to how older people living with frailty make decisions, including decisions about moving to long-term care. The purpose of this study was to explore their needs and the out-of-pocket expenses associated with those needs.

Method: An exploratory mixed method study was conducted, including interviews with five older people identified as frail or pre-frail and seven caregivers identified through purposive sampling, and consultation with stakeholders to obtain cost estimates.

Results: We identified categories of expenses including caregiving, home modifications, property maintenance, heavy cleaning, transportation and social engagement. Findings will be reported as case exemplars: the needs of four participants will be presented, along with estimates of the costs to enable community living for each person.

Conclusions: Older people living with frailty have complex needs that may contribute to financial burden. Proactive planning by individuals and policy makers is needed to support older people living with frailty to live well in the community.

\section{Critical Care's Impact on the Gut Microbiome in Frailty: A Study on Cognitive Outcomes}

E. R. Hawken ${ }^{1,2}$, J. Muscedere ${ }^{3,4}$, J. G. Boyd ${ }^{3}$. ${ }^{1}$ Department Psychiatry, Queen's University, Kingston, ON, Canada; ${ }^{2}$ Department of Biomedical and Molecular Sciences, Queen's University, Kingston, ON, Canada; ${ }^{3}$ Department of Medicine, Queen's University, Kingston, ON, Canada; ${ }^{4}$ Department of Critical Care Medicine, Queens University, Kingston, ON, Canada.

Objective: The project objectives were to determine the feasibility of measuring the gut microbiome in frail and nonfrail individuals admitted to the intensive care unit (ICU) and to ultimately identify a role of the gut microbiome in the development and course of frailty and associated cognitive impairments.

Method: To assess feasibility, pilot data (10 ICU patients and their fecal samples) were collected from FORECAST (Frailty, Outcomes, Recovery and Care Steps of Critically Ill Patients) at three time points (ICU admission, discharge, 6-month follow-up). Two meta-analyses examining relationships between gut microbiome diversity and frailty or cognition were also conducted.

Results: We report recruiting progress of this pilot-portion of FORECAST. Meta-analyses were performed using search criteria restricted to adult human populations, frailty or cognition measures and microbial-diversity of the gut microbiome. Meta-analysis of three identified studies showed a significant negative correlation between diversity and severity of frailty, i.e., that a loss of microbial diversity in the gut is associated 
with increased frailty status. Data has yet to be extracted from the 13 studies identified in the microbiome and cognition meta-analysis.

Conclusions: Identifying a biophysical signal associated with frailty may provide new interventional targets to improve outcomes in frail individuals.

Exploring Understanding of Illness to Improve End-ofLife Communication in Older Adults with Heart Failure and Their Family Caregivers

J. $\operatorname{Im}^{1}$, S. Mak ${ }^{2}$, R. Upshur ${ }^{3}$, L. Steinbreg ${ }^{4}$, K. Kuluski ${ }^{3}$. ${ }^{1}$ Institute of Health Policy, Management and Evaluation, University of Toronto, Toronto, ON, Canada; ${ }^{2}$ Division of Cardiology, Mount Sinai Hospital, Sinai Health System, Toronto, ON, Canada; ${ }^{3}$ Bridgepoint Collaboratory in Research and Innovation, Lunenefeld-Tanenbaum Research Institute, Sinai Health System, Toronto, ON, Canada; ${ }^{4}$ Palliative Care Consult Team, Mount Sinai Hospital, Sinai Health System, Toronto, Ontario, Canada.

Background: End-of-life communication in older adults with heart failure is challenged by clinical uncertainty given the high-risk of morbidity and mortality, and the presence of multimorbidity and frailty. These same challenges underscore the need for earlier end-of-life communication. Understanding of illness is an important barrier of end-oflife communication in patients and caregivers. This study explores how understanding of illness influences engagement in end-of-life discussions.

Methods: Interpretive description qualitative study of older adults with advanced heart failure and caregivers recruited from a sub-specialty heart failure clinic in Ontario, Canada. Fourteen semi-structured interviews were conducted with 19 participants. Interviews were transcribed verbatim and analyzed alongside data collection.

Results: Participants were knowledgeable of the illness management process, yet, did not understand the implications of serious illness despite their multimorbidity and frailty. As a result of this limited understanding, most participants had not engaged in prior end-of-life discussions with family members or clinicians.

Conclusion: Given the burden of multimorbidity and frailty, understanding the implications of serious illness is needed in older adults and caregivers to perceive a need for end-of-life communication. Addressing the consequences of illness in patient care can improve end-of-life communication.
Consideration of Psychosocial Factors in Frailty Screening: A Scoping Review Protocol

J. Van Damme, P. Stolee. University of Waterloo, Waterloo, ON, Canada.

Background: Many definitions of frailty do not include psychosocial factors, despite considerable research on the links between frailty and psychosocial factors such as social isolation. This study will summarize the current state of the literature on how psychosocial factors are considered in frailty screening and interventions and make recommendations for future research.

Methods: A scoping review of the literature is proposed following guidelines by Arskey and O'Malley (2005) and Levac and colleagues (2010). Relevant studies will be chosen from electronic databases focused on social science, health, medicine, and rehabilitation. Data from relevant literature will be extracted, charted, collated, summarized and reported. Interviews with key informants will be used to review and validate findings from the literature review. Interviews will be audio recorded, transcribed verbatim, and analyzed using Braun \& Clarke's (2006) thematic analysis methodology, supported by NVivo software.

Potential Impact: With improved understanding of frailty screening processes, changes can be made to utilize more patient-centered, holistic approaches that maximize health and social outcomes while providing context for future research and policy.

Conclusion: When screening tools and interventions recognize psychosocial as well as bio-medical factors, there may be opportunities to enhance the effectiveness of screening and interventions through more individualized approaches.

\section{Care Provision to the Older Adult Living with Frailty in Primary Health Care Team-Based Models in Ontario: An Exploratory Multiple Case Study}

M-E. Delvin ${ }^{1}$, J. Medves ${ }^{1}$, K. Woddhouse ${ }^{2} .{ }^{1}$ School of Nursing, Queen's University, Kingston, ON, Canada; ${ }^{2}$ Faculty of Engineering, Queen's University, Kingston, ON, Canada.

Background: The older adults living with frailty require model of primary health care that foster collaboration and care integration through multidisciplinary teams. These models are not well understood and their efficacy needs to be articulated. Furthermore, there is a need to comprehend how care is delivered at the patient level and how patients perceive the care received. The purpose of this study is to explore the care provision to older adults living with frailty 
within three distinct team-based models in Ontario and how frailty is identified in each.

Methods: A multiple case study design with nested cases will be used to explore attributes and processes of team-based models of care in Ontario. Three nested cases in each model will be described to provide in-depth understanding of interactions between the patient and the team. Data collection includes surveys, semi-structured interviews, observations and document analyses. Within case and cross case analyses will be conducted for both cases and nested-cases.

Results: Preliminary data reveal the use of various strategies for identifying and assessing frailty by health care professionals within a team-based model.

Conclusion: Frailty identification and assessment is not well defined within a team-based model.

\section{Dementia, Frailty and the Alternate Level of Care Experience}

M. L. Sakamoto. University of British Columbia, Vancouver, BC, Canada.

Background: Alternate Level of Care (ALC) patients tend to be older adults living with dementia waiting for residential care, often remaining in hospital for many weeks resulting in increased frailty and overall functional decline. Overall, more can be done to improve these patients' hospital care. Unfortunately, there is little research that focuses on the care experiences and needs of ALC patients with dementia.

Methods: This proposed qualitative study will utilize Interpretive Description and will take place at a large Western Canadian hospital. Methods will include observation of ALC patients with dementia $(n=10)$ focusing on their care and daily hospital routines (120 hours total), informal conversations with patients, chart reviews, and interviews with family members $(n=10)$ and hospital staff $(n=10)$.

Impact: Thematic analysis of collected data is expected to illuminate the care experiences and needs of ALC patients with dementia, and provide the basis for recommendations to improve their hospital care. This study has the potential to bring important and much needed attention to these vulnerable and frail patients' hospital experiences.

Conclusion: In order to improve hospital care for ALC patients living with dementia, it is crucial to first take the steps to fully understand their care experiences and specific needs.
Caring Ahead: Developing a Questionnaire to Measure Caregiver Death Preparedness in Dementia

P. Durepos ${ }^{1}$, J. Ploeg ${ }^{1}$, N. Akhtar-Danesh ${ }^{1}$, T. Sussman ${ }^{3}$, H. Punia $^{2}$, S. Kaasalainen ${ }^{1}$. 'School of Nursing, McMaster University, Hamilton, ON, Canada; ${ }^{2}$ Hamilton Health Sciences Corporation, Hamilton, ON, Canada; ${ }^{3}$ School of Social Work, McGill University, Montreal, QC, Canada.

Background: A palliative approach to care is recommended to improve quality-of-life for persons with dementia (PwD) and family caregivers (CG). Guidelines for a palliative approach stress the importance of helping CGs prepare for end-of-life. Feelings of preparedness predict $C G$ well-being in bereavement, and should be optimized in dementia where CGs experience higher rates of complicated grief than other CGs. Advance Care Planning (ACP) aims to promote preparedness, however there is no holistic way to measure ACP effectiveness or preparedness. Hence, we propose to develop and evaluate a multi-dimensional questionnaire to be titled Caring Ahead, to measure CG death preparedness in dementia and ACP effectiveness.

Methods: This study will use a sequential mixed methods design (quan + QUAL). Interviews will be held with bereaved CGs to identify core concepts of preparedness and generate questionnaire items. Next, a Delphi-survey will be conducted with an expert panel to revise a draft questionnaire. Lastly, questionnaire psychometrics will be evaluated with 200 CGs from long-term care.

Potential Impact: Through this study a multi-dimensional questionnaire based upon the Theoretical Framework of Preparedness for End-of-Life (Hebert and colleagues, 2006) will be developed. This study will expand understanding of preparedness, allow stakeholders to evaluate and later design ACP interventions.

\section{GERAS Dancing for Cognition and Exercise (DANCE): A Feasibility Study}

P. Hewston ${ }^{1}$, C. Kennedy ${ }^{1}$, D. Merom ${ }^{2}$, G. Ioannidis ${ }^{1}$, C. Patterson $^{1}$, S. Marr ${ }^{1}$, J. Lee ${ }^{1}$, R. Sztramko ${ }^{1}$, L. Trainor ${ }^{3}$, A. Grenier ${ }^{4}$, M. Woolhouse ${ }^{5}$, A. Papaioannou ${ }^{1}$. ${ }^{1}$ Department of Medicine, McMaster University, Hamilton, ON, Canada; ${ }^{2}$ Health Science, Western Sydney University, Penrith, NSW, Australia; ${ }^{3}$ Department of Psychology, Neuroscience and Behaviour, McMaster University, Hamilton, ON, Canada; ${ }^{4}$ Department of Health, Aging and Society, McMaster University, Hamilton, ON, Canada; ${ }^{5}$ Faculty of Humanities, McMaster University, Hamilton, ON, Canada.

Background: To evaluate the feasibility of GERAS DANCE for older adults with early cognitive and mobility impairments. 
Methods: The progressive dance curricula was delivered for 15-weeks (1-hr class; twice weekly) in older adults (aged: 60 years of age and older) with early cognitive (MoCA score (standard deviation $)=21.77(4.00))$ and mobility $(96 \%$ were pre-frail $/$ frail as indicated on the FRIED) impairments. All participants were able to follow basic instructions and move independently. Feasibility outcomes included: recruitment/retention, adherence, participant satisfaction and social connectivity.

Results: Twenty-five older adults with early cognitive and mobility impairments were recruited from a geriatric outpatient clinic or within the community. A total of $20 / 25(80 \%)$ participants completed the study. Average class attendance was $72 \%$ and self-reported homework adherence "most-days / everyday" was $83 \%$. Stepwise progression in the dance curricula was observed with increases in motor complexity and balance demands. Over $90 \%$ of participants rated GERAS DANCE as excellent and $50 \%$ of participants connected outside of class-time.

Discussion: GERAS DANCE is a feasible and enjoyable program for older adults with early cognitive and mobility impairments. GERAS DANCE curricula grading (duration; sequence; instructions) and performance skills (motor complexity of rhythm and coordination; balance demands) appear to be appropriately tailored for this population.

\section{Minutes of Aerobic Exercise Benefits Executive Function in Older Adults}

A.F.M. Petrella, M. Heath. School of Kinesiology, University of Western Ontario, London, ON, Canada.

Background: By 2031, frontal lobe executive disorders will affect 937,000 Canadians with older adults ( $>55$ years) living with frailty being particularly vulnerable. Fortunately, exercise benefits executive function in cognitively healthy and older adults with self-reported cognitive complaints (sCC). It is, however, unclear whether 'brief' exercise session can improve executive function in older adults and those with sCC. Accordingly, this study had older adults and persons with sCC complete 10-minutes of aerobic exercise at participantspecific intensities.

Methods: Participants (aged 73.72, 50\% female) completed a peak $\mathrm{VO}_{2}$ test to develop participant-specific moderate ( $80 \%$ of lactate threshold [LT]), heavy ( $15 \%$ of LT and peak $\mathrm{VO}_{2}$ difference) and very-heavy (50\% of LT and peak $\mathrm{VO}_{2}$ difference) exercise intensities and participants completed 10-minute aerobic exercise session at each of the aforementioned intensities. Executive function was assessed pre- and post-exercise using the antisaccade task (saccade mirrorsymmetrical to a stimulus).
Results: Both groups demonstrated decreased antisaccade reaction times post-exercise, independent of exercise intensity.

Conclusions: 10-minutes of aerobic activity across a continuum of intensities enhances executive function in older adults and those with $\mathrm{sCC}$. Antisaccades provide the sensitivity necessary to immediately detect post-exercise executive function changes in older adults.

\section{Storage and Disposal of Opioids: Medication Challenges with End-of-Life Care in the Home}

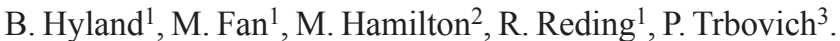
${ }^{1}$ Office of Research and Innovation, North York General Hospital, Toronto, ON, Canada; ${ }^{2}$ Institute for Safe Medication Practices Canada, Toronto, ON, Canada; ${ }^{3}$ Institute of Health Policy, Management and Evaluation, University of Toronto, Toronto, ON, Canada.

Background: End-of-life care often requires evidence-based use of opioids. Improperly securing or disposing of medications, including opioids, in patients' homes has resulted in medication accidents. The objectives were 1) identify preferred practices for storage and disposal of opioids during end-of-life care in the home, 2) develop educational materials for patients/families, and health-care providers.

Methodology: A situation assessment included: a literature review; thematic analysis of 155 out of 1285 articles meeting inclusion criteria and 20 multi-disciplinary stakeholder interviews; and process mapping. An information card was developed with usability testing and research ethics board approval.

Results: Analysis revealed the need to improve patient/family education with the increasing demand for home-based end-oflife care. Complementary preferred practices were identified:

1. Homecare service providers retrieve unused medications.

2. Pharmacists providing in-home medication reviews retrieve unused medications.

3. Patients/families return medications; they can contact www.healthsteward.ca to identify participating pharmacies.

The information card developed supports practice \#3.

Conclusions: The information card can be used by healthcare providers when counselling end-of-life care patients and families. Project partners have committed to supporting its dissemination and knowledge translation.

Caring for the Caregiver: Use of Employee and Family Assistance Programs within NB Nursing Homes 
D. M. O'Reilly ${ }^{1}$, E. A. Read ${ }^{1}$, S. O'Donnell ${ }^{1}$, P. Bruning $^{2}$, J. Donovan ${ }^{3} .{ }^{1}$ Faculty of Nursing, University of New Brunswick, Fredericton, NB, Canada; ${ }^{2}$ Faculty of Business Administration, University of New Brunswick, Fredericton, NB, Canada; ${ }^{3}$ Research Coordinator, York Care Centre, Fredericton, NB, Canada.

Background: Nursing home workers play a vital role in caring for the frail elderly and are particularly vulnerable to job burnout and poor mental health due to their demanding jobs. Yet, Employee and Family Assistance Programs (EFAPs) provided to support employees coping with work and life demands are often underutilized. The purpose of this follow-up study is to understand participants' experiences and perceptions of their EFAP, with emphasis on factors that inhibit and facilitate EFAP use within nursing homes.

Methods: Qualitative data will be collected through oneon-one interviews with 10-15 nursing home employees and 10-15 managers in New Brunswick. Data will be transcribed and analyzed to discover themes. Two interview guides were constructed specific to employee/manager interviews. Employee interview guides focus on depression, and awareness/use of EFAP, while manager interviews focus more on supports for employees.

Results: We anticipate that participants will provide valuable insights into EFAP use and perceived usefulness of the program. Once identified, these factors can be targeted to improve use of this valuable employee resource.

Conclusions: Workplace EFAPs are an underutilized resource among nursing home employees in NB. This research will advance our understanding of this important phenomenon.

\section{Skeletal Muscle Radiodensity and Cross-Sectional Area Predicts Functional Outcomes in Elderly Patients Undergoing Emergency Surgery}

G. Ali ${ }^{1}$, A. Anoveros-Barrera ${ }^{2}$, A. Dunichand-Hoedl ${ }^{2}$, R. G. Khadaroo $^{3}$, V. C. Mazurak ${ }^{2}$, V. E. Baracos ${ }^{1} .{ }^{1}$ Department of Oncology, Faculty of Medicine and Dentistry, University of Alberta, Edmonton, AB, Canada; ${ }^{2}$ Department of Agricultural, Food \& Nutritional Science, Faculty of Agriculture Life and Environmental Sciences, University of Alberta, Edmonton, AB, Canada; ${ }^{3}$ Department of Surgery, Faculty of Medicine and Dentistry, University of Alberta, Edmonton, $\mathrm{AB}$, Canada.

Background: Hospitalized elderly individuals are especially vulnerable to losses of muscle mass and function. Reduced muscle mass and radiodensity may be associated with frailty/ functional outcomes.
Methods: In this retrospective cohort study, elderly female emergency surgery patients $(\mathrm{n}=34)>65 \mathrm{yr}$ at the University of Alberta Hospital, were assessed (Clinical Frailty Scale (CFS), EQ5D Index, timed up \& go (TUG) and sit-to-stand (STS) tests). Muscle cross sectional area $\left(\mathrm{CSA} ; \mathrm{cm}^{2}\right)$ and radiodensity (SMR; Hounsfield units) were quantified in axial lumbar CT images. Pearson's correlation coefficient was calculated.

Results: Patients were aged $76.1 \pm 8.4(\mathrm{SD})$ yr, BMI 27.7 $\pm 6.5 \mathrm{~kg} / \mathrm{m}^{2}$, CFS $3.68 \pm 1.53$ (range 1-6). CFS was found to associate with CSA $(r=-0.356, p=0.039)$ and SMR $(r=-$ $0.370, p=0.031$ ). STS was found to positively correlate with $\operatorname{CSA}(r=0.532, p=0.006)$ and SMR $(r=0.404, p=0.045)$. Neither CSA nor SMR were found to associate with EQ5D or TUG. No correlation was found between CSA and SMR. BMI was not related to any measured outcome.

Conclusions: Elderly emergency surgery patients with higher values of SMR and CSA were less frail, and scored more positively for functional outcomes. SMR and CSA may be useful prognostic measures for functional outcomes of elderly emergency surgery patients.

\section{Prevalence of Sarcopenia Among Active Older Adults and Older Adults Undergoing Joint Replacement}

G. Coletta ${ }^{1}$, S. Phillips ${ }^{2,3}$, J. Jakubowski ${ }^{2}$, S. Atkinson ${ }^{4}$, A. Papaioannou ${ }^{5,6}$, J. M. Pritchard ${ }^{2,6,7}$. ${ }^{1}$ School of Interdisciplinary Sciences, McMaster University, Hamilton, ON, Canada; ${ }^{2}$ Department of Kinesiology, McMaster University, Hamilton, ON, Canada; ${ }^{3}$ McMaster Physical Activity Centre of Excellence, McMaster University, Hamilton, ON, Canada; ${ }^{4}$ Department of Pediatrics, McMaster University, Hamilton, ON, Canada; ${ }^{5}$ Department of Medicine, Division of Geriatric Medicine, McMaster University, Hamilton, ON, Canada; ${ }^{6} \mathrm{Ge}-$ riatric Education and Research in Aging Sciences (GERAS) Centre, Hamilton Health Sciences, Hamilton, Ontario, Canada; ${ }^{7}$ School of Interdisciplinary Sciences, McMaster University, Hamilton, Ontario, Canada.

Background: The Foundation for the National Institutes of Health (FNIH) uses validated cut-points in muscle mass and strength to define sarcopenia, which is an associate of weakness along with frailty. The study was conducted to determine the prevalence of low muscle mass and weakness in active older adults and older adults undergoing total joint replacement (TJR).

Methods: This cross-sectional study included adults $\geq 65$ years of age who were active or who were scheduled to undergo TJR (hip or knee) at the Juravinski Clinic (Hamilton, ON). Handgrip strength and standardized appendicular lean mass (ALM/body mass index) were assessed according to the 
FNIH Sarcopenia Project. Weak grip strength was classified as $<31.83 \mathrm{~kg}$ for men and $<19.99 \mathrm{~kg}$ for women, and muscle mass was considered low if standardized ALM was $<0.725 \mathrm{~m}^{2}$ for men and $<0.591 \mathrm{~m}^{2}$ for women.

Results: This study included 52 participants (37 active[ $62 \%$ male], 15 TJR[40\% male]) who were 75 (6) years of age. There were 17/37 (46\%) and 3/15 (20\%) participants classified as weak from active and TJR groups, respectively ( $p=$ $0.081)$. There were $5 / 37(14 \%)$ and $6 / 15(40 \%)$ participants classified as having low muscle mass from active and TJR groups, respectively ( $p=0.034)$.

Conclusion: Our analysis suggests low muscle mass, and not weakness, seems to be more common in older adults undergoing TJR.

Factors Contributing to a Burden-Free Death: A CrossSectional Study in Western Canadian Nursing Homes

G. E. Werner, M. Hoben, C. A. Estabrooks. Faculty of Nursing, University of Alberta, Edmonton, AB, Canada.

Background: Nursing home (NH) residents are among our frailest and most vulnerable populations. Quality end of life (EoL) care ensuring minimum burden (e.g., pain, dyspnea, depression) is of essence in NHs. However, research persistently has identified high rates of EoL burden in NHs. We address a critical research gap: The Prevalence and associated factors of a burden-free death in NHs.

Methods: We utilized data from 4,906 NH residents who died 04/2014-09/2015 in a representative sample of 91 urban NHs in Western Canada. Burdensome symptoms and resident characteristics came from RAI-MDS 2.0 records. NH characteristics came from validated surveys. We ran generalized linear mixed models with symptom burden as dependent variable and added resident and $\mathrm{NH}$ characteristics identified in a focused literature review as covariates.

Results: 7.6\% (N=374) residents had no burdensome symptoms at the EOL. Adjusted for NH characteristics, older age increased the odds of a burden-free death. Increasing physical and cognitive impairment, higher health instability, cardiac dysrhythmia, gastrointestinal disease, and inappropriate use of antipsychotics decreased the odds of a burden-free death.

Conclusions: Understanding factors that contribute to a burden-free death is paramount to improving quality of EOL and death. Modifiable factors like inappropriate use of antipsychotics are particularly critical.
Impact of Geriatric Emergency Medicine Nurses on Patient Care in the Emergency Department

H. R. Leaker ${ }^{1}$, J. Holroyd-Leduc ${ }^{2}$, L. Fox ${ }^{1} .{ }^{1}$ Faculty of Nursing, University of Calgary, Calgary, AB, Canada; ${ }^{2}$ Cumming School of Medicine, University of Calgary, Calgary, AB, Canada.

Background: Geriatric Emergency Medicine (GEM) nurses help identify and link frail older adults to appropriate community services, to better meet their health and functional needs. The aim of this systematic review is to determine if GEM nurses working within emergency departments (EDs) is associated with positive patient and/or health system outcomes.

Methods: A literature search of MEDLINE, EMBASE, CINAHL, and the Cochrane Databases was conducted to identify English language experimental or quasi-experimental studies reporting on patient and/or health systems outcomes associated with GEM nurses. The search was supplemented by a grey literature search using the CADTH Grey Matters tool. Two reviewers independently reviewed all citations and relevant articles for inclusion. Study quality will be assessed using GRADE and data will be extracted from included articles using a standardized abstraction form.

Results: 4461 articles were retrieved from the database search. 187 articles are currently being reviewed in full text. Data from included articles will be summarized to determine the impact of GEM nurse interventions on care of the frail elderly in the ED.

Conclusions: These results will help to determine if the GEM nurse model should be implemented across Canadian EDs.

\section{An Exploration of End-of-Life Care for Older Adults Identifying as Lesbian, Gay, Bisexual, or Transgender}

J. Smallbone ${ }^{1}$, A. Stinchcombe ${ }^{2,3}$, K. Wilson ${ }^{1}$, K. KortesMiller ${ }^{4,5} .{ }^{1}$ Department of Family Relations and Applied Nutrition, University of Guelph, Guelph, ON, Canada; ${ }^{2}$ School of Psychology, University of Ottawa, Ottawa, ON, Canada; ${ }^{3}$ Department of Health Sciences, Lakehead University, Thunder Bay, ON, Canada; ${ }^{4}$ School of Social Work, Lakehead University, Thunder Bay, ON, Canada; ${ }^{5}$ Centre for Education and Research on Aging and Health, Lakehead University, Thunder Bay, ON, Canada.

Older LGBT adults encounter unique obstacles within the Canadian health-care system when accessing end-of-life care; furthermore, these barriers are exasperated in those unable to self-advocate due to physical vulnerability, and those with reduced capability to adapt to stress. This poster presents findings 
from qualitative research that focuses on identifying said barriers in order to give health-care providers the knowledge necessary to provide competent care to older LGBT adults and reduce discrepancies in quality of care received during end-of-life. Focus groups were held in three separate Ontario cities in order to understand hopes and fears around end-of-life and the lived experience of aging as a LGBT individual from $(n=23)$ adults aged 57 to 78 . Thematic analysis was used to code the data and identify core themes from the focus group participants. Key themes included social support, fear of discrimination, cultural competence in the health-care system, and the intersection of age, sexual orientation, and frailty. Specifically, it is noted that frail adults were less likely to selfadvocate. Recommendations for future practice and policy are presented, notably increasing education in LGBT-competency and LGBT-specific health issues to improve quality of care and end-of-life care for aging individuals.

Statin Use Associated with Calcification, Insulin Resistance, Hypoandrogenism and Cognitive Decline

K. J. Rees-Milton ${ }^{1}$, M. Hulbert ${ }^{1}$, M. E. Turner ${ }^{2}$, C. Berger ${ }^{5}$, T. P. Anastassiades ${ }^{1}$, W. M. Hopman ${ }^{3}$, M. A. Adams ${ }^{2}$, W. L. Powley ${ }^{4}$, R. M. Holden ${ }^{1,2} .{ }^{1}$ Department of Medicine, Queen's University, Kingston, ON, Canada; ${ }^{2}$ Department of Biomedical and Molecular Science, Queen's University, Kingston, ON, Canada; ${ }^{3}$ Clinical Research Center and Department of Public Health Sciences, Queen's University, Kingston, ON, Canada; ${ }^{4}$ School of Computing, Queen's University, Kingston, ON, Canada; ${ }^{5}$ Research Institute of the McGill University Health Centre, Montreal, QC, Canada.

Background: Statins inhibit the mevalonate pathway that is critical to the generation of vitamin $\mathrm{K}_{2}$ in peripheral tissues. Vitamin $\mathrm{K}_{2}$ is involved in glucose homeostasis, vascular calcification, inhibition and prevention of apoptosis. We hypothesized that statin use would be associated with more calcification and insulin resistance (HOMA).

Methods: Statin use and type was assessed in CaMoS participants. Sample size varied from 39 to 604. Abdominal aortic calcification (AAC) was assessed on lateral spine X-ray and insulin resistance was measured by HOMA in non-diabetic subjects at year 10 .

Results: In a stratified Wilcoxon 2 sample test using ranked propensity score adjustment to control for potential confounders, statin users had higher AAC $(\operatorname{Pr}>|\mathrm{Z}|<0.05)$. Using a general linear model, statin use was associated with higher levels of HOMA after stratified propensity score adjustment $[\beta=2.51$, $(4.57,1.48), p<0.01]$. Hydrophilic statin users had the highest HOMA compared to non-statin users $[\beta=8.51,(3.16,22.91)$, $p<0.0001)]$ in a general linear model after adjustment for sex.
Conclusions: Statins, widely prescribed drugs to lower cholesterol, may have unintended consequences related to peripheral vitamin $\mathrm{K}_{2}$ deficiency that could be relevant in aging.

\section{A Community Choir to Facilitate Psychosocial and Cog- nitive Health for Caregivers and Persons with Dementia}

K. Grewal ${ }^{1}$, D. Sheets ${ }^{2}$, A. P. Smith ${ }^{3}$, M. Trites ${ }^{1}$, M. Kennedy ${ }^{4}$, S. MacDonald ${ }^{1} .{ }^{1}$ Department of Psychology, University of Victoria, Victoria, BC, Canada; ${ }^{2}$ Department of Nursing University of Victoria, Victoria, BC, Canada; ${ }^{3}$ Department of Sociology, University of Victoria, Victoria, BC, Canada; ${ }^{4}$ Department of Music, University of Victoria, Victoria, BC, Canada.

Background: Dementias cause progressive neurodegeneration, impacting psychosocial function and increasing cognitive frailty. Non-pharmacological interventions highlight community choirs as one method for addressing dementia-related symptoms. Persons with dementia (PwD) and caregivers benefit through social engagement and mitigating extraneous burden. In this investigation, we examine the benefits of choir participation on changes in psychosocial and cognitive function for PwD and their caregivers.

Method: At baseline, $\mathrm{PwD}(\mathrm{n}=14 ; 66.6 \%$ female) and their informal caregivers $(\mathrm{n}=14 ; 69 \%$ female) were communitydwelling older adults $\left(\mathrm{m}_{\text {age }}=72.92 \mathrm{yrs}, \mathrm{SD}=10.04\right)$. Data from the first of three planned cohorts were gathered through a longitudinal burst design: 4 assessments spanning 3.5 months. Assessments included markers of health, cognition, depression, quality of life, and neuropsychological function.

Results: Multilevel modelling was employed to index changes pursuant to choir participation. For $\mathrm{PwD}$, preliminary findings identified significant episodic memory improvement $(+0.27$ words/assessment, $p<0.05$ ) with a similar trend observed for MMSE gains $(p=0.055)$. Caregivers exhibited significant reduction in depressive symptoms on the PHQ-9 (-1.1 units/ assessment, $p<0.001$ ), with corresponding gains in episodic memory $(+1.27$ words/assessment, $p<0.01)$.

Conclusions: Choirs are an inexpensive and socially-focused intervention that could have far-reaching implications, reducing health-care costs and enhancing quality of life for PwD and their caregivers.

Perceptions of a Home-Based Multi-Faceted Program for Frailty-Risk Seniors

L. Sivarajah ${ }^{1}$, L. Lamarche ${ }^{2}$, C. Kennedy ${ }^{1}$, P. Hewston1, A. Giangregorio ${ }^{1}$, S. Radcliffe ${ }^{1}$, G. Ioannidi ${ }^{1}$, A. Negm ${ }^{1}$, A. 
Papaioannou ${ }^{1} .{ }^{1}$ Geriatric Education and Research in Aging Sciences (GERAS) Centre, St Peter's Hospital, Hamilton, ON, Canada; ${ }^{2}$ Department of Family Medicine, McMaster University, Hamilton, ON, Canada.

Background: Understanding participant perceptions of programs is essential for their adaptability and sustainability, something not always assessed in program effectiveness research. This study explored participant perceptions of a 6-month multifaceted home-based program encompassing exercise, nutrition, medication management and social supports that targeted frailty in community-dwelling seniors.

Methods: Participants were clients of a primary care program designed to foster healthy aging run at two family health teams, and identified as early frailty. Post-program, semistructured interviews were conducted with 15 participants (mean age $=80.6, \mathrm{SD}=8.2$ ). Inductive coding was performed independently by two researchers and a thematic analysis was conducted.

Results: Four main themes emerged: general program, nutrition, social activities, and exercise. Overall, participants appreciated staff support, the health promotion focus and age-appropriateness. Exercise and nutrition interventions led to positive lifestyle changes, and educated participants about their importance to well-being. Barriers to exercise adherence included exercise difficulty and poor health. Dislikes of the nutrition component included travel time to dietitians and inapplicable recommendations to certain health conditions. Many did not see the need to engage in community programs, as they were already well-connected. Participants stated motivated individuals would be ideal program clients.

Conclusions: Overall, participants had positive program perceptions. Findings may inform how to best foster program sustainability.

\section{Measuring Cardiac Dysfunction via Frailty Index in Rat Models}

M. S. Connolly ${ }^{1}$, J. M. Klein ${ }^{2}$, J. S. Huber ${ }^{2}$, S. Safaraz ${ }^{2}$, A. J. Foster $^{2}$, J. A. Simpson ${ }^{2}$, K.R. Brunt ${ }^{3} .{ }^{1}$ Dalhousie University, Halifax, NS, Canada; ${ }^{2}$ Department of Human Health and Nutritional Sciences, University of Guelph, Guelph, ON, Canada; ${ }^{3}$ Department of Pharmacology, Dalhousie University, Halifax, NS, Canada.

Background: Frail patients have worse outcomes than nonfrail patients of the same age. Frailty is a heightened vulnerable state characterized by a decreased capacity to react to biological stressors and results in reduced physical ability. Frailty Indexes (FIs) quantify frailty by accounting for deficits, preferably over time, and enabling mortality risk prediction with potential to better treat vulnerable patients. Currently, no FI effectively models cardiac dysfunction and its mortality risk with treatment.

Methods: Male spontaneously hypertensive rats (SHR; $n=24)$, between 12-32 months old, were randomly assigned a daily angiotensin receptor blocker dose $(15 \mathrm{mg} / \mathrm{kg})$ and were evaluated using a 34-criteria FI. Hemodynamics was determined by echocardiogram and invasive catheterization.

Results: Our FI was found to better predict cardiac dysfunction than chronological age. FI scores and contractility were strongly correlated $\left(\mathrm{dP} / \mathrm{dtmax} \mathrm{R}^{2}=0.31, p=0.01\right.$ and $\mathrm{dP} / \mathrm{dtmin}$ $\mathrm{R}^{2}=0.13, p=0.015$ ). FI scores negatively correlated with hypertension $\left(\mathrm{LVPmax} \mathrm{R}^{2}=0.242, p=0.045\right)$.

Conclusions: Our results demonstrate that FI is an effective prognostic tool for determining cardiac dysfunction. FI usage during treatment may be assist in the monitoring of the effectiveness of both the direct target of intervention and overall treatment efficacy.

\section{The Prognostic Value of CT-Determined Muscle CSA to Identify Frailty States in Critically III Patients}

M. D. Elfassy, L. Munshi. Interdepartmental Division of Critical Care Medicine, Mount Sinai Hospital, Toronto, ON, Canada.

Background: Acute respiratory failure (ARF) is one of the most common causes of admission to an intensive care unit (ICU) for patients with Hematologic Malignancies (HM). This population has a high mortality rate and impaired functional outcomes. Recently, Computed Tomography (CT)-measured muscle Cross-Sectional Area (CSA) has been shown to act as a surrogate for frailty and prognostic outcomes. Our objective is to determine if there is an association between CT- CSA and outcomes in patients with HM and ARF.

Methods: We are performing a retrospective cohort study of ICU patients with ARF and HM. For those who have undergone a CT thorax within 1 month prior to ICU admission, we will evaluate the association between CT CSA measured by the Slice-O-Matic software and ICU outcomes (ventilator-free days at 60 days, ICU and hospital mortality, and functional outcomes following discharge).

Impact/Conclusion: With the majority of patients admitted to the ICU with HM and ARF undergoing CT thorax, CT CSA offers the potential to be a cost-effective, non-invasive, and objective tool to identify different frailty states prior to critical illness. We anticipate CT CSA will be independently 
associated with our outcomes of interest, and aid in clinical decision making, prognostication during an admission, and project recovery.

\section{Use of Proton-Pump Inhibitors in Elderly Hospitalized Patients: An Interdisciplinary Audit}

N. Mehta ${ }^{1}$, F. Martinez Guasch ${ }^{2}$, C. Kamen ${ }^{1}$, S. Shah ${ }^{3}$, L. Burry $^{3}$, C. Soong ${ }^{4,5}$, S. Mehta ${ }^{3} .{ }^{1}$ Faculty of Medicine, University of Toronto, Toronto ON, Canada; ${ }^{2}$ College of Health and Rehabilitation Sciences Boston University, Boston MA, USA; ${ }^{3}$ Sinai Health System; Interdepartmental Division of Critical Care Medicine, University of Toronto, Toronto, ON, Canada; ${ }^{4}$ Division of General Internal Medicine, Sinai Health System, Toronto, ON, Canada; ${ }^{5}$ Institute of Health Policy Management and Evaluation, University of Toronto, Toronto, ON, Canada.

Background: Inappropriate use of Proton Pump Inhibitors (PPI) increases the risk of problematic polypharmacy among frail older adults.

Methods: Retrospective review of patients $\geq 65$ years admitted to the ICU or Acute Care for the Elderly (ACE) unit (0912/2017) who were receiving PPI at admission, or had PPI initiated in hospital. We recorded the prevalence/ indication for pre-hospital and in-hospital PPIs, and whether PPIs were de-prescribed at discharge.

Results: Overall, 89 (29.8\%) of 299 patients (mean age 78 \pm 9 ) were receiving PPI at hospital admission; for gastroesophageal reflux disease (GERD) $(\mathrm{n}=27)$, ulcer/past gastrointestinal bleed $(\mathrm{GIB})(\mathrm{n}=12)$, other $(\mathrm{n}=13)$, and unclear indication $(\mathrm{n}=36) .74$ patients survived to discharge. In surviving ICU patients, $13 \%$ had home PPI de-prescribed at discharge, compared to $28 \%$ in the ACE unit $(p=.002)$. An additional 24/138 ICU and 19/161 ACE patients were newly started on PPI in-hospital ( $17.4 \%$ vs. $11.8 \%, p=.022)$, for GIB ( $\mathrm{n}=19)$, GERD $(n=3)$, other $(n=4)$ and unclear indication $(n=17)$. Of new starts, $8 \%$ ICU and 26\% ACE unit patients were deprescribed PPI at discharge $(p=.07)$.

Conclusion: One-third of elderly patients are receiving PPIs on hospital admission, and a notable percent are newly started in hospital. There is often unclear documentation of indication, raising concerns of potentially inappropriate prescribing.

Falls on Geriatric Hospital Units: What Patient Characteristics are the Culprits?

S. McKay ${ }^{1}$, L. Yetman ${ }^{2}$, J. Slayter ${ }^{2}$, A. McCollum ${ }^{2}$, C. A. McGibbon ${ }^{1}$, P. Jarrett ${ }^{2}$, B. Robinson ${ }^{2}$, A. Kolyvas ${ }^{1}$, R.
McCloskey $^{1}$, S. Gionet ${ }^{2}$, E. Scheme ${ }^{1}$, B. Harris ${ }^{2} .{ }^{1}$ University of New Brunswick, Fredericton, NB, Canada; ${ }^{2}$ Horizon Health Network, Fredericton, NB, Canada.

Background: The risk for falls in older adults is significant during in-patient hospital admissions. Associated consequences of a fall, especially in frail older adults, may be dire for the patients and families. The financial burden to the Canadian health-care system for falls and fall-related injuries in older adults is an estimated $\$ 2$ billion dollars annually. (1) This study explored patient characteristics of fallers versus non-fallers.

Methods: Sixty participants were selected from a larger study using a pseudo-random sampling strategy. A retrospective chart review of three-years of patient fall data was conducted to explore intrinsic and extrinsic patient characteristics of fallers versus non-fallers. The likelihood of falling and becoming a repeat faller was examined to identify interventions that could potentially reduce falls in an in-patient setting. Data extraction included variables such as: medications, co-morbidities, lab values, and clinical documentation.

Impact: Results from this study will expose the patterns and interactions of identified factors and help prevent falls in older adults.

1. Public Health Agency of Canada. Seniors' Falls in Canada: Second Report. Ottawa, ON: PHAC;2014. Retrieved from https://www.canada.ca/en/public-health/services/healthpromotion/aging-seniors/publications/publications-generalpublic/seniors-falls-canada-second-report.html

Developing and Implementing a Frailty Index in the Cardiac Surgery Population for Medical Decision-Making

T. R. D’Aoust ${ }^{1}$, T. Shao', R. Egan' ${ }^{2}$, J. G. Muscedere ${ }^{3}$, B. Milne $^{4}$, M. Fitzpatrick ${ }^{5}$, P. Yingwei Peng ${ }^{6}$, J. Parlow ${ }^{4}$, A. P. Johnson ${ }^{1}$. ${ }^{1}$ Department of Public Health Sciences, Queen's University, Kingston, ON, Canada; ${ }^{2}$ Faculty of Health Sciences/Health Quality Programs, Queen's University, Kingston, ON, Canada; ${ }^{3}$ Department of Critical Care Medicine, Queen's University, Kingston, ON, Canada; ${ }^{4}$ Department of Anesthesiology \& Perioperative Medicine, Queen's University, Kingston, ON, Canada; ${ }^{5}$ Department of Respiratory and Critical Care, Queen's University, Kingston, ON, Canada; ${ }^{6}$ Department of Cancer Care \& Epidemiology, Queen's University, Kingston, ON, Canada.

Background: Cardiac surgery is a common high-cost procedure in Ontario, and patients are older and sicker than in the past. Administrative-level frailty indices have shown ability 
to predict patient outcomes. However, there has been limited work on the use of frailty indices in the cardiac population, and for medical decision-making. The goal of this research was to investigate the predictive ability of a frailty index for cardiac patients and to develop a knowledge-to-action plan.

Methods: Initially, an explorative interview with a key informant was conducted at an Ontario hospital to investigate the local application of frailty indices. The interview was thematically coded and analyzed. Concurrently, a frailty index was developed using administrative-level data to predict outcomes in cardiac surgery patients.
Results: The newly developed frailty index demonstrated ability to predict outcomes in cardiac surgery patients. The key informant suggested using frailty indices to improve medical decision-making through a "patient-engagement tool". A knowledge-to-action plan was proposed to guide the development of a tool informing treatment options and risks.

Conclusion: An administrative-level frailty index demonstrated predictive ability in the cardiac population, and through a patient-engagement tool has the potential to empower patients with their medical decision-making. The proposed knowledge-to-action plan can help guide the implementation. 